\title{
Measuring Stellar Limb Darkening by Gravitational Microlensing
}

\author{
David Heyrovskýn ${ }^{1,2}$
}

\begin{abstract}
Observations of microlensing transit events can be used to measure the limb darkening of the lensed star. We discuss the advantages and drawbacks of several microlensing light curve inversion methods. The method of choice in this work is inversion by means of decomposition of the stellar surface brightness profile. We construct an ideal basis by principal component analysis of brightness profiles obtained from model atmosphere calculations. Limb darkening approximations using such a basis are superior to those using standard power-law limb darkening laws. We perform a full analysis of simulated single-lens microlensing transit events including a detailed error analysis of the method. In realistic events with a low impact parameter the brightness profile of the source can be recovered with a relative accuracy of $2 \%$ from the center of the source disk to 0.9 of the disk radius. We show that in the particular case of the observed MACHO Alert 95-30 event the intrinsic complex variability of the lensed red giant hinders efforts to recover its surface features.
\end{abstract}

Subject headings: gravitational lensing - methods: data analysis — stars: atmospheres

\section{INTRODUCTION}

Our current knowledge of the intrinsic physical properties of stars is largely based on stellar atmosphere models (e.g., Gray 1992). Ever more advanced models are computed for a broad range of stellar types, taking into consideration a range of physical and chemical processes expected to occur in the star's atmosphere.

However, for the vast majority of observable non-variable stars the predictions of these models can be confronted with a single observable quantity - the integrated full-disk spectrum. It follows that any two physically different atmosphere models which predict similar

\footnotetext{
${ }^{1}$ Harvard-Smithsonian Center for Astrophysics, 60 Garden St., Cambridge, MA 02138; dheyrovsky@cfa.harvard.edu

${ }^{2}$ Na Štáhlavce 6, 16000 Praha 6, Czech Republic
} 
integrated spectra cannot be observationally distinguished. More specifically, any observationally "verified" model may have a center-to-limb variation of the spectrum (or limb darkening, in terms of broad-band photometry) very different from the actual star and thus be unphysical, yielding wrong physical parameters of the star. This inherent problem can be overcome by testing atmosphere models in cases or events when the limb darkening of the observed star can be measured.

Until recently the only stars other than the Sun providing the opportunity to directly study their surfaces were the components of eclipsing binaries. Measuring their limb darkening profiles from eclipse light curves proved to be no easy task, due to the degeneracy with other fitted parameters (Grygar, Cooper, \& Jurkevich 1972). Even obtaining useful constraints on the value of the linear limb darkening parameter requires high quality light curves (Popper 1984; Popper et al. 1985).

Extra-solar planets occulting their parent stars are analogous to eclipsing binaries with small secondaries. High precision observations of these scarce systems can also be used for measuring the parent star limb darkening. This was recently demonstrated in the case of HD 209458, the first observed extra-solar occulting system (Jha et al. 2000; Deeg, Garrido, \& Claret 2001; Brown et al. 2001).

The surfaces of nearby giant stars can be resolved by interferometry. Developments in stellar interferometry have led to an advance from measuring stellar radii (Mozurkewich et al. 1991) to detecting the effect of limb darkening (Quirrenbach et al. 1996) to limb darkening measurement for $\alpha$ Ori (Burns et al. 1997) ${ }^{3}$. Hajian et al. (1998) points out the main difficulty of such measurements - signatures of differences between limb darkening profiles become detectable only beyond the first zero of the visibility function. At these high spatial frequencies the fringe visibilities are low, and thus difficult to measure with sufficient precision. Moreover, as shown by Jacob et al. (2000), simultaneous fitting of the stellar radius and limb darkening is complicated by degeneracy. These and other studies indicate that while the use of interferometry for constraining the limb darkening of the source is promising, the accuracy will not reach the level achievable for eclipsing binaries in the near future.

In this work we concentrate on another method - measuring limb darkening from observations of source-transit microlensing events. Galactic gravitational microlensing occurs when a foreground massive object such as a dim star (hereafter "lens") approaches the line of sight to a background star ("source"). Due to gravitational deflection of light by the

\footnotetext{
${ }^{3}$ This nearby supergiant was also recently imaged directly using the Hubble Space Telescope (Uitenbroek, Dupree, \& Gilliland 1998).
} 
lens, the flux from the source is temporarily amplified as the lens passes in the foreground (Paczyński 1996). Currently operating microlensing surveys have already detected over 500 such events toward the rich stellar fields of the Galactic bulge and the Magellanic Clouds. In case the lens directly transits the source star, the light curve of the event will be affected by its limb darkening profile (Witt 1995; Valls-Gabaud 1995; Loeb \& Sasselov 1995). Observations of one such transit event, MACHO Alert 95-30, were reported by Alcock et al. (1997). By the time of writing, limb darkening parameters have been measured also in four binarylens caustic-crossing events: MACHO 97-BLG-28 (Albrow et al. 1999a), MACHO 98-SMC-1 (Afonso et al. 2000) ${ }^{4}$, MACHO 97-BLG-41 (Albrow et al. 2000) and OGLE-1999-BUL-23 (Albrow et al. 2001). ${ }^{5}$

Microlensing thus presents a unique new possibility for studying the surfaces of distant stars (Valls-Gabaud 1995; Sasselov 1996). As discussed in §3, several approaches have been taken by other authors to extract the limb darkening profile from microlensing light curves, with encouraging results. Here we present another inversion strategy providing the most accurate and stable results so far. We achieve this by decomposing the profile using a basis constructed by principal component analysis of a set of actual model atmosphere profiles.

In the following presentation, $\S 2$ contains the basic description of single point-mass lens microlensing of a stellar source. In $\S 3$ we discuss several methods of inverting the microlensing light curve to obtain the surface brightness profile of the source. Specifically, in $\S 3.3$ we provide the basic equations for inversion by brightness profile decomposition. Using model atmosphere calculations and principal component analysis of the model profiles, we determine an ideal basis for brightness decomposition in $\S 4$. The main results are presented in $§ 5$, in which we analyze simulated light curves, simultaneously recovering the lensing parameters and the brightness profile of the source. We perform a detailed error analysis and compute the inversion accuracy for a source with unknown limb darkening. We compare the results with those obtained by fitting the linear limb darkening law, applied in all five published events (an additional square-root coefficient was fitted in MACHO 97-BLG-28). The analysis of the observed MACHO Alert 95-30 event light curves is described in $\S 6$. Limb darkening measurement prospects are discussed in $\S 7$, followed by a summary of the main results in $\S 8$. Two appendices are added, the first describing broadband chromaticity of microlensing light curves, the second discussing the model limb darkening profiles and their fits.

\footnotetext{
${ }^{4}$ This particular event yielded the first and thus far unique case of limb darkening measurement for an A star from a metal-poor population.

${ }^{5}$ Preliminary limb darkening measurement was reported in a fifth event, EROS BLG-2000-5 (An et al. 2002), during the review of this paper.
} 


\section{GRAVITATIONAL MICROLENSING LIGHT CURVES}

Throughout this work we describe the source and lensing geometry in terms of angular displacements in the plane of the sky, using the angular radius of the lensed star as a (projected) distance unit in this plane. ${ }^{6}$ We concentrate here on the case of a spotless source star, with a symmetric surface brightness profile $B(r)$. Microlensing of a spotted source is discussed elsewhere (Heyrovský \& Sasselov 2000).

A point-mass lens located at a projected distance $l$ from the center of the disk of a background star increases the observed flux from this source star to a value

$$
F(l)=\int_{0}^{1} B(r) \mathcal{A}(l, r) r d r,
$$

where $r$ is the distance from the center of the star, and the surface brightness is measured in appropriate units. The angle-integrated amplification $\mathcal{A}(l, r)$ is defined by

$$
\mathcal{A}(l, r)=\int_{0}^{2 \pi} A_{0}\left(\sqrt{l^{2}-2 r l \cos \phi+r^{2}}\right) d \phi,
$$

where $\phi$ is the (source-centered) polar angle between a point on the stellar disk at a distance $r$ and the lens at a distance $l$. The lensing amplification factor $A_{0}(x)$ for a point source at a separation $x$ from the lens is

$$
A_{0}(x)=\frac{x^{2}+2 \epsilon^{2}}{x \sqrt{x^{2}+4 \epsilon^{2}}}
$$

here $\epsilon$ is the angular Einstein radius of the lens (Paczyński 1996) in units of lensed star radius. The integral in equation (2) can be directly solved in terms of elliptic integrals as follows:

$$
\begin{aligned}
\mathcal{A}(l, r)=\frac{4}{(l+r) \sqrt{(l-r)^{2}+4 \epsilon^{2}}} & {\left[2 \epsilon^{2} K\left(\frac{4 \epsilon}{l+r} \sqrt{\frac{l r}{(l-r)^{2}+4 \epsilon^{2}}}\right)+\right.} \\
& \left.+(l-r)^{2} \Pi\left(\frac{4 l r}{(l+r)^{2}}, \frac{4 \epsilon}{l+r} \sqrt{\frac{l r}{(l-r)^{2}+4 \epsilon^{2}}}\right)\right] .
\end{aligned}
$$

\footnotetext{
${ }^{6}$ Using the angular Einstein radius as a unit (traditional microlensing notation) is not convenient in this work concentrating on the source star structure. The conversions between our parameters and the parameters in the standardized notation proposed by Gould (2000) are $l=u \theta_{E} / \theta_{*}, \epsilon=\theta_{E} / \theta_{*}, p=u_{0} \theta_{E} / \theta_{*}$ and $t_{*}=t_{E} \theta_{*} / \theta_{E}$.
} 
We use standard notation for the complete elliptic integrals of the first $(K)$ and third (П) kind. In the latter we follow the sign convention used by Byrd \& Friedman $(1971)^{7}$, $\Pi\left(\alpha^{2}, k\right)=\int_{0}^{\pi / 2}\left(1-\alpha^{2} \sin ^{2} \theta\right)^{-1}\left(1-k^{2} \sin ^{2} \theta\right)^{-1 / 2} d \theta$.

Notice that $\mathcal{A}(l, r)$ is symmetric in its arguments $l, r$. Also note that $\mathcal{A}(0, r)=2 \pi A_{0}(r)$, as well as $\mathcal{A}(l, 0)=2 \pi A_{0}(l)$. When the lens is far from the source $(l \gg 1, \epsilon)$, the angleintegrated amplification drops to $2 \pi$. In the limit of a strong lens $(\epsilon \gg 1)$, the angleintegrated amplification at the light curve peak $(\epsilon \gg l)$ in equation (1) can be approximated by

$$
\mathcal{A}(l, r) \simeq \frac{4 \epsilon}{l+r} K\left(\frac{2 \sqrt{l r}}{l+r}\right),
$$

an expression linearly dependent on the Einstein radius $\epsilon$. In this work we use the more general expression in equation (4). Figure 1 contains sample plots of the angle-integrated amplification as a function of radial distance $r$ for Einstein radii $\epsilon=1$ and 10 (the latter scaled down by a factor of 10 for comparison), and several lens positions. At the position of the lens $(r=l)$ there is a logarithmic divergence, so that for $r=l+\delta$

$$
\mathcal{A}(l, l+\delta) \simeq \frac{2 \epsilon}{l}\left(1-\frac{\delta}{2 l}\right) \ln \frac{8 \epsilon l}{|\delta| \sqrt{l^{2}+\epsilon^{2}}}+4 \arctan \frac{l}{\epsilon}+\frac{\epsilon\left(2 l^{2}+\epsilon^{2}\right)}{l^{2}\left(l^{2}+\epsilon^{2}\right)} \delta+\mathcal{O}\left(\delta^{2} \ln |\delta|\right)
$$

where $|\delta| \ll 1$. Due to this divergence at the lens position (for $l \leq 1$ ) in the integrand of equation (1), the lens efficiently scans the surface brightness profile of the source star as it moves across the stellar disk. The general microlensing effect on the spectrum of model stellar atmospheres was demonstrated by Heyrovský, Sasselov, \& Loeb (2000, hereafter HSL). We provide a brief discussion of broadband light curve shapes in Appendix A of this work.

\section{LIGHT CURVE INVERSION METHODS}

Photometric observations of microlensing events provide us with sets of observed fluxes $F_{i}$ measured at different times $t_{i}$ with estimated errors $\sigma_{i}(i=1, \ldots n)$. Primary analysis of a transit event yields the main (geometric) lensing parameters: the Einstein radius $\epsilon$, impact parameter $p$, time of closest approach $t_{0}$ and angular source radius crossing time $t_{*}$. The latter three parameters help us mainly to convert the observation times $t_{i}$ to lens positions $l_{i}$, as shown further in equation (29). In the two following sections (through $\S 4$ ), we assume zero blended flux (contribution of unresolved stars) and consider the geometric parameters to be fixed at predetermined values, in order to facilitate the inversion analysis. In $\S 5$ and $\S 6$

\footnotetext{
${ }^{7}$ Press et al. (1992) use an opposite sign for the first parameter.
} 
we proceed to the full event analysis including the effect of blending, and recover the lensing and limb darkening parameters simultaneously.

The aim of this work is to extract the brightness profile $B(r)$ from the observed fluxes $F_{i}$. The mathematical problem is to invert equation (1), classified as a Fredholm integral equation of the first kind. This problem has no universal method of solution. The task is more difficult in this particular case, because of the required high level of accuracy of the result. The aim is not just to detect limb darkening, but to actually measure it. Based on solar observations and model atmosphere calculations, broadband limb darkening profiles in the optical range vary only to a fairly limited degree (see Appendix B), hence the required precision.

There is a number of different numerical approaches to inverting equation (1) that can be tested. Craig \& Brown (1986) provide a good overview of strategies for solving astrophysical inverse problems. In the following two subsections we discuss two methods applied previously by other authors to the microlensing case. The third subsection introduces the method used in this work.

\subsection{Inversion by Discretization}

A straightforward method is to invert equation (1) by discretization, approximating the integral by a sum. For a light curve with $n$ points we can select $n_{D} \leq n$ radial points $r_{j}$ $\left(j=1, \ldots n_{D}\right)$ on the source disk and write

$$
F\left(l_{i}\right)=\int_{0}^{1} B(r) \mathcal{A}\left(l_{i}, r\right) r d r=\sum_{j=1}^{n_{D}} \mathbb{D}_{i j} \breve{B}\left(r_{j}\right)
$$

for each lens position $l_{i}(i=1, \ldots n)$. Here the matrix element $\mathbb{D}_{i j}$ is some approximation of $\int \mathcal{A}\left(l_{i}, r\right) r d r$ over the vicinity of point $r_{j}$.

In order to obtain brightness point estimates $\breve{B}\left(r_{j}\right)$ providing a good fit to the light curve data, we now minimize

$$
\chi^{2}=\sum_{i=1}^{n} \sigma_{i}^{-2}\left[F_{i}-F\left(l_{i}\right)\right]^{2}
$$

Rescaling the matrix to $\widetilde{\mathbb{D}}_{i j} \equiv \mathbb{D}_{i j} / \sigma_{i}$ and the measured fluxes to $\tilde{F}_{i} \equiv F_{i} / \sigma_{i}$, we can write the $\chi^{2}$-minimizing solution explicitly:

$$
\breve{B}\left(r_{j}\right)=\sum_{i=1}^{n} \sum_{k=1}^{n_{D}}\left(\widetilde{\mathbb{D}}^{T} \widetilde{\mathbb{D}}\right)_{j k}^{-1} \widetilde{\mathbb{D}}_{i k} \tilde{F}_{i},
$$


here $\widetilde{\mathbb{D}}^{T}$ denotes the transpose of matrix $\widetilde{\mathbb{D}}$. The errors due to light curve noise for each of the obtained brightness points are given by the standard deviations

$$
\sigma_{\breve{B}_{j}}=\sqrt{(\widetilde{\mathbb{D}} T \widetilde{\mathbb{D}})_{j j}^{-1}}
$$

In comparison with the other methods discussed subsequently, this direct approach has the advantage of not making explicit assumptions about the shape of the brightness profile.

Gaudi \& Gould (1999) presented an error analysis of this method ${ }^{8}$, demonstrating that the surface brightness profile can be recovered only for the outer radial part of the stellar disk directly transited by the lens. The profile of the inner part, at radii smaller than the impact parameter of the lensing event, cannot be accurately reconstructed.

The general failure of microlensing light curve inversion in this region can be explained as follows. For any light curve point $l_{i}>0$, the matrix element $\mathbb{D}_{i j} \rightarrow 0$ for inner disk points $r_{j} \rightarrow 0$ by definition. Hence, in the case of a non-zero impact parameter the associated value $\breve{B}\left(r_{j}\right)$ has negligible weight in all the sums given by equation (7), and thus is weakly constrained by the data. Alternatively, this failure can be understood by noting the low microlensing sensitivity to stellar spots located off the projected lens trajectory (e.g., a circular spot at the disk center; see Heyrovský \& Sasselov 2000).

Returning specifically to inversion by discretization, some of its intrinsic problems are directly apparent from equation $(7)$. For transit points $\left(l_{i}<1\right)$ the integrand is divergent, as demonstrated by equation (6). Obtaining an adequate approximation of such an integral by a sum requires a fairly high number of terms, i.e., an even higher number of transit light curve points. Even in the case of negligible light curve noise, simulations show that discretization with a limited number of points introduces errors that make the method unstable and the inversion unreliable.

As a further point, straightforward discretization assuming constant surface brightness within annuli of the source disk can introduce additional errors. Unless there is a sufficient number of radial points defining the annuli, this assumption cannot be made particularly near the limb of the source, where the brightness drops rapidly.

The stability of this method can be improved by introducing additional constraints on the solution points $\breve{B}\left(r_{j}\right)$. For example, one might require positiveness of the solution, its monotonic decrease or concavity. Typically these constrain the solution points by a set of linear inequalities. The solution is then found numerically by minimizing $\chi^{2}$ given by equa-

\footnotetext{
${ }^{8}$ Their paper covers the case of binary lensing as well, in the linear caustic crossing regime. Here we discuss their single lens results.
} 
tion (8), while satisfying the imposed inequalities. Bogdanov \& Cherepashchuk (1996) took this approach in the case of non-transit events ${ }^{9}$, seeking a non-negative concave decreasing solution. Unfortunately, they made no estimate of the errors or stability of their results. In a following paper (Bogdanov \& Cherepashchuk 1999), the authors presented their inversion of the MACHO Alert 95-30 light curves, by seeking a non-negative decreasing solution. No stability estimates were made in this work either, moreover the data manipulation is questionable (neglecting observational errors, converting fluxes to amplifications prior to inversion etc.).

Even the constrained method has its implicit drawbacks. Either the unconstrained solution given by equation (9) automatically satisfies the desired constraints, or the constrained solution has to satisfy at least one equality instead of the required inequality. This is a direct consequence of the minimized function $\chi^{2}$ being quadratic and positive definite in its parameters $\breve{B}\left(r_{j}\right)$. Such a function has only one global minimum, given by the unconstrained solution (9), and no other local minima. If this minimum does not lie within the constrained region of parameter space, the constrained solution lies on the boundary of the region. A requirement of positiveness therefore forces the constrained solution to have at least one zero point, a requirement of monotonic decrease forces a constant section of the solution, concavity forces a linear section, etc. A combination of constraints forces an equality in at least one constraint. Even if the method provides reasonable-looking solutions, there is no guarantee these approximate the actual brightness profile of the source. Without an error analysis based on simulations the results cannot be trusted.

\subsection{Backus-Gilbert Method}

The Backus-Gilbert method (e.g., Craig \& Brown 1986) takes quite a different approach to inverting equation (1). It sets out to determine an approximation of the brightness at some point $r_{0}$ as a linear combination of all the observed fluxes,

$$
\bar{B}\left(r_{0}\right)=\sum_{i=1}^{n} \mu_{i}\left(r_{0}\right) F_{i}
$$

\footnotetext{
${ }^{9}$ This choice is not ideal, as non-transit events contain little information about the surface brightness distribution of the source (see HSL and Appendix A).
} 
where $\mu_{i}\left(r_{0}\right)$ are suitable coefficients. Substituting the theoretical expectations $F\left(l_{i}\right)$ for the fluxes from equation (1), we find

$$
\bar{B}\left(r_{0}\right) \simeq \int_{0}^{1} S\left(r, r_{0}\right) B(r) d r
$$

where the introduced spread function $S\left(r, r_{0}\right)$ is a linear combination of the integral kernels corresponding to all lens positions in equation (1), using the same coefficients,

$$
S\left(r, r_{0}\right) \equiv \sum_{i=1}^{n} \mu_{i}\left(r_{0}\right) \mathcal{A}\left(l_{i}, r\right) r
$$

The spread function should be normalized so that a uniform brightness profile is recovered identically by equation (12), namely $\int_{0}^{1} S\left(r, r_{0}\right) d r=1$. The same equation suggests it is desirable to select the coefficients $\mu_{i}\left(r_{0}\right)$ so that the spread function approaches the delta function $\delta\left(r-r_{0}\right)$. This narrowness (high resolution) of the spread function is sought by requiring for example

$$
\int_{0}^{1}\left(r-r_{0}\right)^{2} S^{2}\left(r, r_{0}\right) d r=\text { Min. }
$$

The final constraint imposed on the coefficients limits the error magnification,

$$
\sigma_{\bar{B}\left(r_{0}\right)}^{2}=\sum_{i=1}^{n} \mu_{i}^{2}\left(r_{0}\right) \sigma_{i}^{2} \leq M \sum_{i=1}^{n} \sigma_{i}^{2}
$$

the constant $M$ is a measure of the statistical error tolerance. The set of coefficients $\mu_{i}\left(r_{0}\right)$ can now be easily found analytically by minimizing equation (14) using the definition from equation (13) together with the normalization condition and the error magnification constraint. We then compute the brightness point $\bar{B}\left(r_{0}\right)$ from equation (11) and its error due to light curve noise from equation (15).

The method tends to be laborious - the procedure has to be repeated for each desired point $\bar{B}\left(r_{0}\right)$ individually. Another drawback lies in having the free parameter $M$. Decreasing its value on one hand increases the stability of the result towards light curve noise, but on the other hand decreases the resolution (yields a broader spread function). As a result, some user-defined optimization is necessary. It should be noted that there is no explicit requirement that the obtained solution provide a good fit to the light curve data. The result may be stable towards light curve noise and yet be incorrect due to systematic effects. This should be checked independently. 
Preliminary reports on testing the Backus-Gilbert method were presented by Hendry et al. (1998) with a recent update by Gray \& Coleman (2000). In both cases their presented sample result is for a lens with an Einstein radius $\epsilon=1$ and a non-transit impact parameter $p=1$. It should be noted that for these parameters the surface brightness signature on the light curve is very weak (see HSL and Appendix A), in fact lower than the $2 \%$ noise added to the simulated light curve in Hendry et al. (1998) ${ }^{10}$. Using a larger Einstein radius (which is also more realistic) should provide better results. At radii $0.6 \lesssim r \lesssim 0.9$ the recovered brightness approaches the original profile, though there is a systematic discrepancy in the residuals. Between 0.5 and 0.6 the obtained profile peaks and starts decreasing (unphysically) towards smaller radii, reaching a $\sim-18 \%$ deviation at $r=0.05$ (even worse, $\sim-25 \%$ in Gray \& Coleman 2000). As a consequence, the recovered profile has a lower integrated flux, and thus will not provide a good fit to the observed light curve. The authors state that the deviation for the region $r<0.6$ does not improve with reduced light curve noise. The inversion turns unacceptably noisy for 10 or less light curve points according to the authors. It is apparent that in this case the actual inversion error is determined by systematic effects rather than by statistical errors. The accuracy is too low for the results to be of much use for measuring the limb darkening of the source.

The Backus-Gilbert technique seems to be poorly suited for microlensing light curve inversion in general. In the case of non-transit events, all the kernels on the right-hand side of equation (13) are very similar functions, increasing monotonically from the source center towards the limb. It is difficult to construct a spread function narrowly peaked at any selected point on the disk by a linear combination of such terms. At any rate, this would require at least some of the coefficients $\mu_{i}\left(r_{0}\right)$ to be negative. However, subtracting fluxes in equation (11) only increases the effects of noise. The problem in recovering the brightness profile of the inner disk is also apparent: at $r=0$ all the kernels are identically equal to zero. Getting a spread function peaked in this region would require high values of the coefficients, which would increase its amplitude at the limb even more.

In the case of transit lens positions, the corresponding kernels in equation (13) are divergent at these points. Getting a spread function at least effectively narrow-peaked at a particular point by a weighted combination of functions divergent at different points seems to be difficult. It remains to be seen whether and how the Backus-Gilbert technique could be modified to be useful for microlensing light curve inversion.

\footnotetext{
${ }^{10}$ Gray \& Coleman (2000) show that the results do not change qualitatively if the light curve noise is varied from $0.2 \%$ to $10 \%$, which suggests the result is an artifact of the method rather than a measurement of the source profile.
} 


\subsection{Limb Darkening Decomposition}

We can avoid a number of problems encountered by the previous methods by decomposing the brightness profile into a linear combination of $n_{C} \leq n$ basis functions $f_{j}(r)$,

$$
B(r) \simeq \hat{B}(r)=\sum_{j=1}^{n_{C}} \alpha_{j} f_{j}(r)
$$

We can now rewrite equation (1),

$$
F\left(l_{i}\right)=\int_{0}^{1} B(r) \mathcal{A}\left(l_{i}, r\right) r d r \simeq \sum_{j=1}^{n_{C}} \mathbb{C}_{i j} \alpha_{j}
$$

where the matrix elements $\mathbb{C}_{i j}$ are defined by

$$
\mathbb{C}_{i j} \equiv \int_{0}^{1} \mathcal{A}\left(l_{i}, r\right) f_{j}(r) r d r
$$

and can be therefore computed to arbitrary precision. We now use equation (17) to minimize $\chi^{2}$ given by equation (8), obtaining the best-fit coefficients

$$
\alpha_{j}=\sum_{i=1}^{n} \sum_{k=1}^{n_{C}}\left(\widetilde{\mathbb{C}}^{T} \widetilde{\mathbb{C}}\right)_{j k}^{-1} \widetilde{\mathbb{C}}_{i k} \tilde{F}_{i}
$$

As previously, we rescaled the matrix elements to $\widetilde{\mathbb{C}}_{i j} \equiv \mathbb{C}_{i j} / \sigma_{i}$. The standard deviations of the obtained coefficients due to light curve noise are

$$
\sigma_{\alpha_{j}}=\sqrt{(\widetilde{\mathbb{C}} T \widetilde{\mathbb{C}})_{j j}^{-1}}
$$

This method is mathematically closely related to inversion by discretization. However, unlike in the case of discretization, the involved integrals can be computed to arbitrary precision and therefore do not contribute to the inversion error. The quality of this method is given by the accuracy of the decomposition in equation (16), which is the only introduced approximation. In principle, with a sufficiently high number of decomposition terms the range of brightness profile shapes is restricted only negligibly. However, with a limited number of terms this method is clearly model-dependent, unlike discretization. In the following section we proceed to construct a basis $f_{j}(r)$ suitable for inverting broadband light curves. 


\section{SELECTION OF DECOMPOSITION BASIS}

To test the decomposition method, we generate simulated microlensing light curves using sample brightness profiles obtained directly from model atmosphere computations (see HSL and Heyrovský \& Loeb 1997 for more details). We explore a principally four-dimensional parameter space, setting the Einstein radius $\epsilon$, impact parameter $p$ (position of the closest light curve point here), number of light curve points $n$ (in most cases only transit points are used), and relative noise level $\eta$. We add noise to the simulated light curve by setting

$$
F_{i}=F\left(l_{i}\right)\left[1+\eta_{i}\right],
$$

where $\eta_{i}(i=1, \ldots n)$ is a Gaussian-distributed random number with zero mean and standard deviation $\eta$. Hence $\eta F\left(l_{i}\right)$ represents the error $\sigma_{i}$.

For a given basis $f_{j}(r), j=1, \ldots n_{C}$ we evaluate the quality of the inversion $(B / \hat{B}-1)$ and compare it with results obtained for the standard limb darkening (hereafter SLD) basis

$$
g_{1}(r)=1, \quad g_{j}(r)=-\left[1-\sqrt{1-r^{2}}\right]^{j-1} \text { for } j>1 .
$$

In this basis two terms $\left(n_{C}=2\right)$ correspond to the usual linear limb darkening law, three to the quadratic law, one to uniform brightness. Four out of the five published microlensing limb darkening measurements mentioned in $\S 1$ used the linear law. Albrow et al. (1999a) measured an additional term $\left(1-\sqrt[4]{1-r^{2}}\right.$, square-root law) in MACHO 97-BLG-28.

In the following subsection we illustrate some general features and problems of this method on the case of an analytical basis. In $\S 4.2$ we derive an ideal basis by principal component analysis of a set of brightness profiles obtained from realistic model atmosphere calculations.

\subsection{Polynomial Basis}

We first try to set $f_{j}(r)=P_{j}(r)$, where $P_{j}(r)$ is a polynomial satisfying the requirement $d P_{j} / d r(0)=0, P_{1}(r)$ is a constant, and $P_{j}(r)$ is of degree $j$ for $j>1 .{ }^{11}$ Any brightness profile can be well approximated using several terms of such a basis. In the following we briefly summarize the main results of inversion tests.

The brightness profile close to the limb is generally poorly constrained by light curve data. For practical purposes we concentrate on the inversion accuracy for the interior region $r \lesssim 0.9$ of the stellar disk.

\footnotetext{
${ }^{11}$ The inversion result $\hat{B}(r)$ is independent of the explicit form of the basis satisfying these criteria.
} 
As one would expect from the microlensing chromaticity curve (see Appendix A), the lower the impact parameter, the better the inversion. While non-transit points with small errors can help improve the inversion accuracy, transit points are necessary for achieving an accuracy better than $10 \%$. The inversion quality is independent of the Einstein radius for $\epsilon \gtrsim 3$, it deteriorates slowly for $\epsilon<3$. This is in agreement with the microlensing sensitivity to surface structure, which doesn't increase much for $\epsilon \gtrsim 3$ while for smaller values ${ }^{12}$ it drops to zero, as illustrated in Appendix A.

The inversion accuracy rapidly deteriorates when using more than a couple of basis terms. Moreover, the number of terms necessary for obtaining the best inversion is not correlated with the changing $\chi^{2} /$ (degree of freedom). As one cannot directly judge the quality of the inversion by the quality of the light curve fit, it is necessary to predetermine an ideal number of terms to extract from a light curve with given parameters. Generally, we find it is possible to extract 4 polynomial terms for a noise level of $0.1 \%, 3$ terms for a currently achievable level of $0.5 \%$, and merely 2 terms (i.e., a parabola) for more common noise levels of $1 \%$ and $2 \%$. In the SLD case, only 2 terms (i.e., standard linear limb darkening) are reliably obtainable in general.

Overall, the SLD inversions tend to be superior to the tested polynomial inversions. However, the quality of the SLD results depends on the underlying brightness profile $B(r)$. The results can be systematically worse in profiles poorly described by linear limb darkening. The tested polynomial basis is not ideal either. It describes a too general class of functions, so that it is not possible to extract a sufficient number of terms to provide a reliable and useful light curve inversion. From these tests we conclude we need a basis significantly more constrained than general polynomials, but capable of describing already in two terms a broader range of limb darkening profiles than does standard linear limb darkening.

\subsection{Basis Constructed by Principal Component Analysis}

For constructing an adequate basis we start out with $B V R I$ profiles obtained from eight computed red giant model atmospheres (see Appendix B and Fig. 2), using filter response functions provided by Bessell (1990). Our goal is to find a basis giving the best gradual (single-term, two-term, ...) approximation to these $n_{M}=32$ different brightness profiles $B_{i}(r), i=1, \ldots n_{M}$. We can obtain the first normalized component of the basis

\footnotetext{
${ }^{12}$ As noted in HSL, for practical purposes the lower cutoff is $\epsilon \simeq 0.4$. Events with smaller Einstein radii are too weak (maximum amplification $\lesssim 1.34$ ) to trigger an alert in most current surveys.
} 
$\left(\int_{0}^{1} f_{1}^{2}(x) d x=1\right)$ by minimizing the total integrated square residual

$$
\mathcal{R}(f)=\sum_{i=1}^{n_{M}} \int_{0}^{1}\left\{b_{i}(x)-\left[\int_{0}^{1} b_{i}(y) f(y) d y\right] f(x)\right\}^{2} d x,
$$

where $b_{i}(r)$ are the model profiles, suitably normalized. The inner integral is the coefficient approximating $b_{i}(x)$ by $f(x)$. The minimization can be performed by the calculus of variations, leading to an equation of the form

$$
\int_{0}^{1} \sum_{i=1}^{n_{M}} b_{i}(x) b_{i}(y) f(y) d y=\lambda_{f} f(x)
$$

We can see that the sought function $f_{1}(r)$ is an eigenfunction of a positive semidefinite integral operator with a symmetric kernel. It can be shown not only that $f_{1}(r)$ corresponds to the highest eigenvalue $\lambda_{f_{1}}$, but also that all the further components of the basis can be obtained by extracting normalized eigenfunctions with successively lower eigenvalues ${ }^{13}$. The total integrated square residual for a $k$-component basis is

$$
\mathcal{R}\left(f_{1}, \ldots f_{k}\right)=\sum_{i=1}^{n_{M}} \int_{0}^{1}\left\{b_{i}(x)-\sum_{j=1}^{k}\left[\int_{0}^{1} b_{i}(y) f_{j}(y) d y\right] f_{j}(x)\right\}^{2} d x
$$

its minimization leads to equation (24) for each of the components independently, thanks to their orthogonality. The eigenvalue for the $j$-th component is equal to the decrement of the residual $\mathcal{R}$ due to the addition of the $j$-th component to the basis,

$$
\lambda_{f_{j}}=\sum_{i=1}^{n_{M}}\left[\int_{0}^{1} b_{i}(x) f_{j}(x) d x\right]^{2} .
$$

By substituting the constructed eigenfunction basis into equation (25), we obtain the actual value of the total residual,

$$
\mathcal{R}\left(f_{1}, \ldots f_{k}\right)=\sum_{i=1}^{n_{M}} \int_{0}^{1} b_{i}^{2}(x) d x-\sum_{j=1}^{k} \lambda_{f_{j}}
$$

\footnotetext{
${ }^{13}$ As a bonus, orthogonality of the eigenfunctions, $\int_{0}^{1} f_{i}(x) f_{j}(x) d x=\delta_{i j}$, is guaranteed, because the operator in equation (24) is Hermitian.
} 
From this expression we can see that the normalization of the brightness profiles which gives each an equal weight in this analysis is

$$
b_{i}(r)=\left[\int_{0}^{1} B_{i}^{2}(y) d y\right]^{-1 / 2} B_{i}(r)
$$

and consequently $\mathcal{R}\left(f_{1}, \ldots f_{k}\right)=n_{M}-\sum_{j=1}^{k} \lambda_{f_{j}}$. If there are $n_{I}$ linearly independent profiles among $B_{i}(r)$, there are exactly $n_{I}$ positive eigenvalues - all the rest are zero. Their sum is $n_{M}$, hence they leave a zero residual. In most cases based on non-trivial model atmosphere calculations (such as in this case), all $B_{i}(r)$ are linearly independent and $n_{I}=n_{M}$.

The approach sketched above is a special case of principal component analysis (e.g., Preisendorfer 1988; Jolliffe 1986). The eigenfunction problem involves solving the homogeneous Fredholm integral equation (24), which can be easily achieved numerically by iteration. The first three basis functions obtained by principal component analysis of our 32 model profiles are presented in Figure 3.

We demonstrate the superiority of the first two components of the basis over linear limb darkening in Appendix B, for the model profiles as well as for data from optical observations of the Sun. This result suggests that although the basis was constructed from brightness profiles of cool red giants, it can be used for a broader class of sources. On the other hand, similar bases can be constructed using sets of model atmospheres of different stellar types. The main reasons for using red giants in this work are their intrinsic brightness (observational advantage of achieving better photometry) and large size (advantage of getting better coverage of the transit). In addition, red giants are numerous in the Galactic bulge fields observed by the microlensing survey teams. Finally, the only well-documented single lens microlensing transit event by the time of writing, MACHO Alert 95-30 (Alcock et al. 1997), involved a red giant source.

Initial inversion tests with the basis obtained by principal component analysis (hereafter PCA) and fixed lensing parameters as in the previous subsection show that the systematic errors plaguing the SLD inversions are significantly reduced. The obtained results are generally more accurate and less biased. However, it is necessary to test PCA inversion in the full light curve analysis, without knowing the lensing parameters a priori.

\section{FULL INVERSION OF SIMULATED LIGHT CURVES}

In order to demonstrate the reliability and quality of the described PCA inversion, we test the method for a representative range of lensing parameter combinations and source 
brightness profiles. We estimate the statistical error by inverting a number of noisy light curves for each parameter combination, and the systematic error from the results obtained for different brightness profiles. For comparison, we perform the same procedure using linear limb darkening (hereafter LLD).

In the following subsection we describe the implementation of the simulations and the light curve inversion algorithm. In $\S 5.2$ we discuss the accuracy of the recovered lensing parameters, and in $\S 5.3$ the obtained limb darkening of the source. Based on these results, we suggest in $\S 5.4$ how to analyze observed light curves in order to accurately recover the limb darkening as well as the lensing parameters.

\subsection{Simulation and Inversion Algorithms}

We generate simulated light curves for combinations of five lensing parameters: Einstein radius $\epsilon$, impact parameter $p$, blend parameter $\beta$ (ratio of flux from unresolved stars to baseline flux $F_{*}(\infty)$ of lensed star), number of points per source diameter crossing time $n_{*}$ (equal to transit point number for small impact parameters), and light curve noise level $\eta$. The two parameters defining the time scale of the simulated event can be kept fixed at arbitrary values, for simplicity we set the source radius crossing time $t_{*}=1$ and the time of closest approach $t_{0}=0$. However, we do fit for both of these parameters in the following light curve analysis. In order to get a reasonable fit for most of the parameters we include non-transit points, setting the total number of points to $n=2 n_{*}$ and distributing them in equal time intervals from roughly $2 t_{*}$ before the peak to $2 t_{*}$ after the peak ${ }^{14}$. The lens positions are given by

$$
l_{i}=\sqrt{p^{2}+\left[\left(t_{i}-t_{0}\right) / t_{*}\right]^{2}}
$$

for $i=1, \ldots n$. We compute the source star fluxes $F_{*}\left(l_{i}\right)$ directly from equation $(1)$ using one of the model brightness profiles (not its approximation) and the angle-integrated amplification from equation (4). We integrate across the transit point divergence using expression (6) and, to match the accuracy of this expansion, a local linear approximation of the brightness profile. To obtain the simulated fluxes $F_{i}$ we add the blend contribution and Gaussian noise $\eta_{i}$ with standard deviation $\eta$ (as in the previous section),

$$
F_{i}=\left[F_{*}\left(l_{i}\right)+\beta F_{*}(\infty)\right]\left[1+\eta_{i}\right] .
$$

The errors are then given by $\sigma_{i}=\eta\left[F_{*}\left(l_{i}\right)+\beta F_{*}(\infty)\right]$.

\footnotetext{
${ }^{14}$ Explicitly, $t_{i}=t_{0}+[(4 i-3) / n-2] t_{*}, i=1, \ldots n$. This distribution is slightly asymmetric around the peak.
} 
The studied parameter space is a Cartesian grid defined by all combinations of values $\epsilon \in\{1,2,5,10\} ; p \in\{0,0.25,0.5,0.75\} ; \beta \in\{0,0.25,0.5\} ; n_{*} \in\{10,20,40\} ;$ and $\eta \in$ $\{0.005,0.01,0.02\}$. As shown in Appendix A, higher values of $\epsilon$ provide the same sensitivity to surface structure as $\epsilon=10$, even though they provide higher overall amplification. For lenses with Einstein radii lower than 1 the sensitivity drops, for even lower values such events would be hard to detect at all, due to their low amplification. Events with impact parameters $p \gtrsim 1$ contain only a weak signature of the surface structure, so that their inversion is useless under realistic noise conditions, as shown in the previous section. The choice of blend parameters is geared toward events with bright (e.g., red giant) source stars, for which the fraction of blended light can be expected to be low. The point numbers are representative and realistic. The $0.5 \%$ noise level has been achieved in several microlensing events, the two other values are more usual.

The model fitted to the light curves is based on equation (17) with additional blended flux $F_{B}$,

$$
F_{M}\left(l_{i}\right)=F_{B}+\alpha_{1} \mathbb{C}_{i 1}\left(\epsilon, l_{i}\right)+\alpha_{2} \mathbb{C}_{i 2}\left(\epsilon, l_{i}\right),
$$

where the matrix elements $\mathbb{C}_{i j}$ are given by equation (18), and the lens positions by equation (29). In this model the asymptotic flux (flux in absence of the lens) from the lensed star is $F_{*}(\infty)=\alpha_{1} C_{01}+\alpha_{2} C_{02}$, where $C_{0 j}=\lim _{l_{i} \rightarrow \infty} \mathbb{C}_{i j}$ for $j=1,2$. These two values are $C_{01} \doteq 2.680, C_{02} \doteq 2.418$ for the PCA basis and $C_{01} \doteq 3.142, C_{02} \doteq-1.047$ for the LLD basis. The value $F_{B} / F_{*}(\infty)$ corresponds to the blend parameter $\beta$ as defined above.

This seven-parameter model is linear in three parameters $\left(F_{B}, \alpha_{1}, \alpha_{2}\right)$, and depends on the remaining four $\left(\epsilon, p, t_{*}, t_{0}\right)$ through $\mathbb{C}_{i j}\left[\epsilon, l_{i}\left(p, t_{*}, t_{0}\right)\right]$. In order to speed up the subsequent computations, it is crucial to precalculate these matrix elements for a sufficiently dense grid of Einstein radii $\epsilon$ and lens positions $l$ using the two basis functions ( $f_{1,2}$ for PCA and $g_{1,2}$ for LLD) and equations (4) and (6), in a manner similar to the source light curve computation described above. Values at intermediate points can be obtained by interpolation.

Due to the form of the dependence on the nonlinear parameters the full inversion problem cannot be solved analytically. Instead we find numerically the best-fit parameters by minimizing

$$
\chi^{2}=\sum_{i=1}^{n} \sigma_{i}^{-2}\left[F_{i}-F_{M}\left(l_{i}\right)\right]^{2} .
$$

However, the numerical search can be limited to only four out of the seven dimensions, due to the presence of three linear parameters (as also noted by Rhie et al. 1999 and Albrow et al. 1999b). For any fixed combination of the four nonlinear parameters, the combination of $\left(F_{B}, \alpha_{1}, \alpha_{2}\right)$ minimizing the $\chi^{2}$ function can be found analytically by solving a set of 
three linear equations. These are the corresponding minimum conditions obtained by differentiating equation (32). It is advantageous to exclude non-physical solutions in advance by requiring for example a non-negative blend, a non-negative brightness profile peaked at the center (see Appendix B), and the total model asymptotic flux being within $\pm \sigma_{0}$ of the baseline flux $F_{0}$ (both constants estimated from the data). These five constraints restrict the linear parameter space to a pentahedron. If the computed minimum (for any fixed set of nonlinear parameters) lies outside this region, the constrained minimum can be found on its surface.

For the minimum search in the remaining four-dimensional parameter space $\left(\epsilon, p, t_{*}, t_{0}\right)$ we used a Monte Carlo technique based on gradual contraction of parameter intervals towards lower values of $\chi^{2}$. This technique was found to be very robust in finding the global minimum.

As the full inversion problem is nonlinear, we inverted 100 noisy light curves for each point of the parameter grid to get a realistic estimate of statistical errors. In order to find the systematic error and bias of the method we tested the 32 model brightness profiles listed in Appendix B. The distribution of the values recovered from different noisy simulations was usually found to be substantially non-Gaussian. Therefore we decided to describe these distributions using the median instead of the mean, and the narrowest interval containing $68.3 \%$ of the recovered values instead of the $\pm \sigma$ standard deviation interval, which contains the same fraction of points in a Gaussian distribution. ${ }^{15}$

With respect to the two photometry-defining parameters $\eta$ and $n_{*}$, a good-quality fit not dominated by systematic errors is indicated also by the linear scaling of the total error with light curve noise and the inverse square root of the number of points $\left(\propto \eta n_{*}^{-1 / 2}\right)$. We tested both these scalings; for the $n_{*}$ scaling we used the results for $\eta=0.005$ in order to suppress the effect of light curve noise.

\subsection{Recovered Lensing Parameters}

Before studying the recovered limb darkening, we check here the accuracy of recovered lensing parameters: namely, Einstein radius $\epsilon$, impact parameter $p$, blend parameter $\beta=F_{B} /\left(\alpha_{1} C_{01}+\alpha_{2} C_{02}\right)$, source radius crossing time $t_{*}$ and closest approach time $t_{0}$. For avoiding confusion we add a subscript ' 0 ' to denote the original values used for the simulations, i.e., $\epsilon_{0}, p_{0}, \beta_{0}, t_{* 0}$ and $t_{00}$. For each point in the original parameter grid, we statistically analyze the full set of $32 \times 100$ recovered parameter values.

\footnotetext{
${ }^{15}$ These two descriptions converge in case the underlying distribution is indeed Gaussian.
} 
We find that the best results for most parameters are obtained in events with high Einstein radius, low impact parameter and low blending. For example, the original parameter combination $\left\{\epsilon_{0}=10 ; p_{0}=0 ; \beta_{0}=0 ; t_{* 0}=1 ; t_{00}=0\right\}$ was recovered using the PCA basis as $\left\{\epsilon=10.11_{-0.19}^{+0.12} ; p=0.016_{-0.016}^{+0.011} ; \beta=\left(1.8_{-1.8}^{+9.8}\right) \times 10^{-3} ; t_{*}=0.9989_{-0.0068}^{+0.0073} ; t_{0}=\left(-0.1_{-1.6}^{+1.4}\right) \times\right.$ $\left.10^{-3}\right\}$ from simulated light curves with $n_{*}=40$ and $\eta=0.01$. As an exception, the impact parameter is recovered most accurately in events with higher impact parameters, transiting closer to the limb of the source star. This is probably due to the weak dependence of the microlensing amplification on the brightness profile in the range $0.65 \lesssim l \lesssim 0.8$ (see Appendices A and B), which in turn implies a lower degeneracy between the remaining fitted parameters (mainly $p$ and $t_{*}$ ).

The closest approach time $t_{0}$ is by far the best-recovered parameter. The accuracy of the recovered Einstein radius and blend parameter decreases slightly in events with $\epsilon_{0} \gtrsim 5$. In such events, better accuracy is obtained by including more distant light curve points in the analysis, as discussed further in $\$ 5$.4. The total error of the PCA results is $\propto \eta n_{*}^{-1 / 2}$ in events with $\epsilon_{0} \gtrsim 1$. The only found exceptions are the impact parameter error when $p_{0}=0$, and the blend parameter error when $\beta_{0}=0$ (both are values at the parameter space boundary).

The LLD results are in most cases qualitatively similar. In a few cases the accuracy is slightly worse and the error scaling with light curve noise and number of points does not follow $\eta n_{*}^{-1 / 2}$ as accurately as in the PCA analysis. Clear systematic differences can be seen in the recovery of the source radius crossing time $t_{*}$. The most interesting difference is found in the general scaling of the errorbars: the errors scale roughly as $\eta^{0.7}$ and $n_{*}^{-0.3}$, quite consistently. A closer study of the numerical results reveals that systematic errors in the $t_{*}$ values recovered using LLD are large - comparable to the statistical errors, hence the different scalings. This means that while the LLD errors for $n_{*}=40$ and $\eta=0.01$ are only slightly larger than the PCA errors, decreasing the light curve noise or increasing the number of points will only boost the relative advantage of PCA analysis over LLD analysis. This problem is most probably caused by LLD generally providing a poor fit to realistic brightness profiles, as demonstrated in Appendix B.

\subsection{Recovered Limb Darkening}

In this subsection we first present the results obtained for three sample input brightness profiles. We then proceed to estimate the actual error of the reconstructed profile for a source with unknown limb darkening by a combined analysis of the simulation results for all 32 tested profiles. 
The shape of the recovered profile is given by the PCA or LLD limb darkening parameter $\kappa=\alpha_{2} / \alpha_{1}$. The accuracy of recovering this parameter can be studied similarly as the lensing parameters in the previous subsection. Of the 32 input model brightness profiles (see Appendix B and Table 1), we present in Figure 4 the results for the most centrally peaked profile (B3750g10), an intermediate one (R3500g05), and the flattest profile (I4000g10). We determine the median and the $68.3 \%$ confidence interval from the 100 noisy light curves computed for each input parameter combination. In addition we plot the minimum and maximum obtained values (none of the simulation results were discarded). The results shown in the figure correspond to $n_{*}=40$ and $\eta=0.01$.

As seen from the results for all profiles and both PCA and LLD fits, limb darkening parameters are most accurately obtained for events with low impact parameter $p_{0}$, although the dependence is weak for $p_{0} \lesssim 0.5$. The accuracy increases only very weakly with higher Einstein radius $\epsilon_{0}$, and is fully independent of blend parameter $\beta_{0}$. These results are encouraging - either limb darkening parameter can be recovered even if the blend or Einstein radius are poorly fitted.

In the case of profile B3750g10, the original LLD parameter value (1.0) lies exactly at the parameter space boundary, as reflected also by the recovered values. The plotted PCA errorbars for the ideal lensing parameter combination $\left(\epsilon_{0}=10 ; p_{0}=0 ; \beta_{0}=0\right)$ are consistent with best-fit PCA limb darkening parameters corresponding to all the $B$ profiles in Table 1. In the LLD case, the errorbars are formally consistent only with the B3750g10 value, due to their nearly zero width. However, this does not mean that the actual profile is recovered better (see Table 1 ).

The results for profile R3500g05 exhibit a nonlinear dependence of the LLD errors on the light curve noise $\eta$, indicating non-negligible systematic errors. The LLD medians are clearly correlated with the impact parameter $p_{0}$. The PCA errorbars for the ideal lensing parameter combination are consistent with best-fit PCA limb darkening parameters corresponding to the R3500 and V4000 profiles in Table 1. In the LLD case, the errorbars are consistent with all profiles from R3500 to B3500, including all $V$ profiles.

The recovered LLD medians for profile I4000g10 systematically underestimate the original value. The PCA errorbars for the ideal lensing parameter combination are consistent with best-fit PCA limb darkening parameters corresponding only to the I4000 profiles in Table 1. The same result is obtained in the LLD case. However, note here that no flatter profiles were used (see Fig. 2 and Table 1).

From the general analysis we conclude that the PCA results have the advantage of being uncorrelated with the lensing parameters and having no apparent systematic problems. The 
LLD medians are correlated with $p_{0}$ and sometimes with $\beta_{0}$; the results suffer from systematic errors due to the inadequacy of the LLD approximation. Even in ideal cases, the LLD results are less accurate than the PCA results.

It is worth noting that this test only told us how well a fitted parameter of the actual brightness profile can be recovered from the light curve. The conclusions confirm that the parameter of a worse-fitting model will be recovered worse. The following part of this subsection studies how well is the actual brightness profile of the source recovered.

Using the results for all 32 profiles it is now possible to make an estimate of the accuracy of the inverted brightness profile $\hat{B}(r)$, given by equation (16), for any given combination of input parameters $\left(\epsilon_{0}, p_{0}, \beta_{0}, n_{*}, \eta\right)$ independent of the actual source brightness profile. First we compute the relative brightness profile deviation for each of the $32 \times 100$ inverted light curves,

$$
\delta(r)=\frac{B(r)}{F_{* 0}(\infty)} \frac{F_{*}(\infty)}{\hat{B}(r)}-1=\frac{B(r)}{F_{* 0}(\infty)} \frac{\alpha_{1} C_{01}+\alpha_{2} C_{02}}{\alpha_{1} f_{1}(r)+\alpha_{2} f_{2}(r)}-1
$$

here $F_{* 0}(\infty)$ is the asymptotic flux of the input model profile $B(r)^{16}$. From this full set of data we determine the total median $\delta_{B}(r)$, which represents the bias of the inversion, and the $68.3 \%$ confidence interval $\left(\delta_{B}-\delta_{L}, \delta_{B}+\delta_{U}\right)$, where $\delta_{L}(r)+\delta_{U}(r)$ is the total error.

To estimate the systematic error, we determine the median deviation for each model profile separately, from its 100 noisy light curves. Analysis of the distribution of these 32 profile-specific medians yields a "median median" $\delta_{M M}(r)$, and the $68.3 \%$ confidence interval $\left(\delta_{M M}-\delta_{S L}, \delta_{M M}+\delta_{S U}\right)$. The value $\delta_{S L}(r)+\delta_{S U}(r)$ is the systematic error, a measure of source-dependence of the inversion accuracy.

From the previous analysis we obtain a best estimate of the brightness profile of the lensed star,

$$
\mathcal{B}(r)=\left[1+\delta_{B}(r) \begin{array}{l}
+\delta_{U}(r) \\
-\delta_{L}(r)
\end{array}\right] \times \hat{B}(r)
$$

If the accuracy of the recovered blend parameter is low, by normalizing this result we still obtain the best estimate of the normalized profile of the star.

A reliable inversion method requires not only a small total error $\delta_{L}+\delta_{U}$, but also negligible bias $\delta_{B} /\left(\delta_{L}+\delta_{U}\right) \rightarrow 0$, so that the inversion result is closest to the best estimate. It is also desirable to have small systematic errors $\left(\delta_{S L}+\delta_{S U}\right) /\left(\delta_{L}+\delta_{U}\right) \ll 1$, so that the

\footnotetext{
${ }^{16}$ The obtained profiles $\hat{B}(r)$ are normalized to the total source flux in order to avoid uncertainties introduced by any potential inaccuracy of the fitted blend.
} 
inversion accuracy doesn't depend substantially on the source profile, and the inversion can yield better results with improved photometry. Such a method would also have $\delta_{M M} \simeq \delta_{B}$.

Representative PCA and LLD results of the described analysis are presented in Figure 5 for $\epsilon_{0}=10, \beta_{0}=0, n_{*}=40$ and $\eta=0.01$. The plotted curves include the bias $\delta_{B}$, the total error as the $68.3 \%$ confidence interval $\left(\delta_{B}-\delta_{L}, \delta_{B}+\delta_{U}\right)$, the "median median" $\delta_{M M}$ and the systematic error as the $68.3 \%$ confidence interval $\left(\delta_{M M}-\delta_{S L}, \delta_{M M}+\delta_{S U}\right)$; all are plotted as a function of the position on the stellar disk $r$. The jagged shape of some of the curves is merely due to using only 32 model profiles in the analysis. All the desirable properties mentioned in the previous paragraph can be achieved by PCA inversion, but not by LLD inversion. The PCA results show a negligible bias all the way to the limb of the source, the coincidence of medians $\delta_{M M} \simeq \delta_{B}$, and systematic errors much smaller than total errors - with a single exception in the case of the best-fit point at $r \simeq 0.7$ with an error on the order of $0.5 \%{ }^{17}$. The quality of the inversion improves weakly with higher Einstein radius $\epsilon_{0}$, and is practically independent of the blend $\beta_{0}$ (only in poorly fitted events the stability deteriorates very weakly with increasing $\beta_{0}$ ). The errors scale linearly with light curve noise $\eta$ and decrease as the inverse square root of the number of points $n_{*}$ per source diameter crossing time. The best inversion is achieved for low impact parameter events. However, even at $p_{0}=0.75$ good accuracy can be achieved by obtaining a higher number of good quality transit points. The results in Figure 5 demonstrate the best obtained inversion (for $p_{0}=0$ ) has errors $\lesssim 2 \%$ from the disk center out to $r \simeq 0.9$. These errors can be further reduced by having better than $1 \%$ photometry or more than 40 light curve points per source diameter crossing time.

While the LLD inversion results are not bad either, they do not achieve the quality of the PCA results and do not have the desirable inversion properties. The results are always biased, on average producing flatter profiles. The bias can reach as much as $2 \%$ in the region $r<0.9$ and diverges further closer to the limb. The systematic errors are large and often dominate the total error, particularly in the region $0.5 \lesssim r \lesssim 0.8$. The two medians $\delta_{M M}$ and $\delta_{B}$ differ by as much as $1 \%$. The trends of the results with $\epsilon_{0}, p_{0}$ and $\beta_{0}$ are the same as in the PCA case. The linear scaling of the errors with $\eta$ and their inverse square root scaling with $n_{*}$ occurs in fewer cases and to a lesser extent than in PCA inversion. The quality of the achievable results is limited by the relatively large systematic errors.

\footnotetext{
${ }^{17}$ This point occurs in the results merely due to the fact that the intersection point of the PCA limb darkening model (as discussed in Appendices B and A) lies in the narrow intersection region of the used set of model profiles. This is not the case for the LLD model.
} 


\subsection{Inversion of Observed Light Curves}

In the simulated inversions performed in the previous subsections we only used points near the light curve peak, within \pm 2 source radius crossing times from the closest approach. Light curves of actual microlensing events usually provide data going well beyond this region. To study the effect of more distant points on the recovery of lensing parameters and the limb darkening, we performed simulations including light curve points within \pm 2 Einstein radius crossing times from the closest approach.

The results for $\epsilon_{0} \gtrsim 5$ show a dramatic improvement in the recovered Einstein radii and blend parameters, as mentioned above. In such events impact parameters are recovered better as well, with the exception of the already well recovered $p_{0}=0.75$ which improves only marginally. The distributions of the recovered source radius crossing times have more accurate medians, but the total errorbars remain unchanged. On the other hand, the accuracy of the recovered closest approach time and (more notably) the limb darkening parameter is worse than while fitting only the event peak. Consequently, the total error of the recovered brightness profile at source center increases by $1-3 \%$.

These findings are a natural result of the light curve fitting procedure. While fitting an extended light curve going far beyond the transit region, the $\chi^{2}$ of the fit is altered more by a minute change in the global parameters (e.g., $\epsilon$ and $\beta$ ) than by a more significant change in the limb darkening parameter, which affects only points in the transit region - a small fraction of the total number of light curve points.

High accuracy for the limb darkening of the source as well as the lensing parameters can be achieved by a two-step procedure. In the first step we fit the full light curve, varying all parameters. In the second we fit only the transit region (not more than \pm 2 source radius crossing times from the peak), varying the limb darkening parameter and the closest approach time, keeping the other parameters fixed at values obtained in the previous step. Finally, we check the $\chi^{2}$ value of the overall result.

\section{ANALYSIS OF MACHO ALERT 95-30 LIGHT CURVES}

In this section we analyze the observed light curves of the microlensing source-transit event MACHO Alert 95-30 (Alcock et al. 1997). Altogether seven light curves were measured by the MACHO and Global Microlensing Alert Network (GMAN; Becker et al. 1997) collaborations. The individual light curves were obtained by the MACHO team at Mount Stromlo Observatory (MSO; MACHO B filter - 293 points used, MACHO R filter - 205 points), and

by the GMAN collaboration at Cerro Tololo Inter-American Observatory (hereafter CTIO; 
R - 106 points), Mount John University Observatory (MJUO; R - 41 points), University of Toronto Southern Observatory (UTSO; R - 133 points, V - 127 points) and Wise Observatory (WISE; R - 17 points). Alcock et al. (1997) presented the results of light curve fits using a uniform source model as well as a linearly limb-darkened source with fixed limb darkening parameters based on theoretical expectations. Here we redo the general light curve fitting procedure $^{18}$ to measure the limb darkening profile of the source, as well as to obtain more accurate lensing parameters.

The analysis can be performed in the manner used in the previous section. The only difference is that this case involves a simultaneous inversion of seven light curves. The four nonlinear lensing parameters $\left(\epsilon, p, t_{*}, t_{0}\right)$ are common to all the curves, the linear parameters $\left(F_{B}, \alpha_{1}, \alpha_{2}\right)$ should be determined for each light curve separately. This is necessary because of differences not only in the used filters, but also in the optics, CCDs, point spread functions, data reduction methods etc. Fitting the 25 parameters to the full set of data is straightforward. As described in the previous section, for a given combination of the four nonlinear parameters all the linear parameters can be computed analytically by minimizing the total $\chi^{2}$ function. Errors of the obtained best-fit parameters can be obtained by Monte Carlo simulations. We generate 100 sets of seven noisy light curves using the obtained bestfit parameters, plus the timings and errorbars of the original data. We invert these curves and from the distribution of the obtained values we determine the (non-Gaussian) $68.3 \%$ confidence intervals for each parameter.

Estimation of the baseline parameters $F_{0}$ and $\sigma_{0}$ for the two MACHO light curves revealed a systematic problem. As shown in Figure 6, the baselines exhibit nearly periodic correlated variations. There is an apparent period of $\sim 45$ days, with an amplitude of $\sim 3.5 \%$ in MACHO B and $\sim 2.9 \%$ in MACHO R. Further inspection of the light curves suggests there is additional irregular variability on both longer and shorter timescales. Due to this complex variability, even within the span of 10 days around the light curve peak the intrinsic flux from the source may change by $\sim 3 \%$. As an immediate consequence, this change will obscure the weak chromatic lensing effect close to the source limb (see Appendix A). Moreover, the limb darkening parameters (i.e., the source brightness profiles) can be expected to vary, rendering any inversion questionable in principle. Finally, the obtained lensing parameters will also be inaccurate to a certain degree, due to the source variations in the light curve peak region.

Proceeding formally with the fit, the problems become even more apparent. The $\chi^{2}$

\footnotetext{
${ }^{18}$ The event data were kindly provided by Andy Becker, who re-reduced the CTIO, UTSO and WISE light curves recently.
} 
value for the fit to the original data is substantially and systematically higher than the values obtained for Monte Carlo simulated light curves with the best-fit parameters. Even the residuals of the fit close to the peak show systematic deviations. The relative residuals $F_{i} / F_{M}\left(l_{i}\right)-1$ are plotted in Figure 7 for this part of the light curve. As can be seen, the fairly large residuals in the different light curves are clearly correlated (e.g., note the post-peak changes).

The formal best fit parameters are: Einstein radius $\epsilon=13.91_{-0.08}^{+0.13}$; impact parameter $p=0.7253_{-0.0044}^{+0.0017}$; source radius crossing time $t_{*}=2.5164_{-0.0028}^{+0.0060} d$ and the closest approach time $t_{0}=1321.2359_{-0.0038}^{+0.0012} d$, measured from JD 2,448,623.50 (Alcock et al. 1997). Except for $t_{0}$, these values are slightly different from those obtained by Alcock et al. (1997). Partly this is caused by the greater number of points near the light curve peak in the current set of data (mainly in the UTSO light curves), partly by the more general fitting procedure. The greatest effect, however, is due to source variability. Neither of the fitted models accounts for the effect of an intrinsically irregularly variable source. All the lensing parameters obtained here as well as in Alcock et al. (1997) should therefore be taken with a grain of salt.

The values of the formal best-fit limb darkening parameters are concentrated at the flat-profile boundary of the explored parameter space. This can be seen also from the largely positive residuals during the transit. Moreover, the best-fit limb darkening parameters are mutually physically inconsistent (implying flatter profiles in $V$ than in $R$ etc.). All these features confirm the conclusion that limb darkening measurement from the MACHO Alert 95-30 event is not possible due to the variability of the lensed star. It cannot be ruled out that some of the residuals close to the peak may be due to the lens being binary (Griest \& Safizadeh 1998; Gaudi \& Sackett 2000), source being binary (Griest \& Hu 1992; Han \& Gould 1997; Han \& Jeong 1998) or even due to asymmetric surface features on the source star (Heyrovský \& Sasselov 2000; Bryce \& Hendry 2000). However, attempts to fit any of these models will also be largely hindered by the variability of the source star.

It should come as no surprise that the lensed M4 giant source in MACHO Alert 95-30 is variable. Percy \& Polano (1998) state that "virtually every M giant is variable in brightness", with small-amplitude red variables being two orders of magnitude more common than Miras. Variability larger than $1 \%$ in the $V$ band sets in already in late $\mathrm{K}$ and early $\mathrm{M}$ type giants, and increases further toward late M type giants (Jorissen et al. 1997; Percy \& Parkes 1998). The variability seems to be associated primarily with pulsations (Percy \& Parkes 1998; Jorissen et al. 1997), as suggested also by small-amplitude radial velocity variations measured by Cummings et al. (1998). The variations are not strictly periodic, sometimes they are multimodal or irregular (Percy \& Polano 1998). The periods can be on the order of tens of days down to single days, as recently demonstrated from Hipparcos data by Koen \& Laney (2000). 
These results demonstrate the importance of checking the baseline of source-transit event light curves to detect even small-amplitude variability. Only events in which the change due to intrinsic variability over the event timescale is sufficiently smaller than the expected microlensing chromaticity (see Appendix A) can be used for measuring the limb darkening of the source star. As an exception, if the pulsations are well defined, regular and stable, they can be accounted for a priori in the lensing model, and the lensing effect can be fully separated from the intrinsic variability. MACHO Alert 95-30 unfortunately does not fall into this category of events.

\section{DISCUSSION}

The accuracy of measuring the limb darkening parameter from light curves of eclipsing binaries was studied in detail by Popper (1984). On the basis of light curve simulations Popper concluded that achieving an absolute accuracy of \pm 0.1 in the linear limb darkening parameter requires on the order of 100 light curve points within light curve minima obtained with $0.5 \%$ photometry. As shown in Figure 4, analysis of microlensing transit light curves frequently achieves better accuracy even when using LLD inversion of light curves measured with $1 \%$ photometry. These results are surpassed in quality by PCA inversion, which in fact directly demonstrates the inadequacy of the linear limb darkening model. Going one step further, the results in Figure 5 show that PCA inversion can retrieve realistic brightness profiles (instead of profile fits) with an accuracy higher than $2 \%$ from the center of the stellar disk out to $r=0.9$. Inversion of microlensing light curves thus gives the opportunity to proceed from measuring the limb darkening parameter to measuring the actual limb darkening profile of the source, thanks to the high achievable accuracy and low systematic errors.

Eclipsing binaries have the advantage of having periodic light curves, giving thus frequent opportunities to measure the eclipse minima. Even though microlensing transit events are non-repeating, giving only a single chance to obtain high quality photometry, their analysis can place much better constraints on the limb darkening as well as other properties of the source (HSL, Heyrovský \& Sasselov 2000). Such events can also potentially provide results for a broad range of stellar sources in the currently surveyed Galactic bulge or Magellanic Cloud fields, as microlensing does not depend on the stellar type of the source. The only biases are introduced by the requirement of good photometry (giving an advantage to brighter sources) and a sufficient number of transit points (giving an advantage to longer transits, i.e., larger sources).

The PCA method described in this work on the example of broadband profiles of red 
giants can be simply extended to any other class of sources, narrow-band filters etc. Using a set of brightness profiles obtained from a corresponding class of atmosphere models for the required wavelengths or passbands, an adequate PCA basis can be constructed as described in $§ 4.2$. The first few terms describing a sufficiently broad range of possible brightness profiles can then be used for the light curve analysis.

Observed events with high quality photometry can also be used to discriminate between classes of stellar atmosphere models with different PCA bases. The more suitable class can be distinguished mainly by the pattern of residuals of the light curve fit. The best-fit model should then be checked whether it is in agreement with measured properties of the unamplified source - such as colors, line strengths etc. Microlensing thus provides unique new tests that will contribute to our understanding of stellar atmospheres.

Microlensing transit events are much more frequent for caustics of binary lenses than for single point lenses studied in this work. The reason is that the probability of a caustic transit of the source is usually given by the angular size of the caustic rather than by the angular size of the source. While the source size still determines the duration of the transit, detection of the source entering a caustic gives an opportunity to predict and observe in detail at least the exit from the caustic. Limb darkening parameters have already been measured for four caustic-crossing events in this way, three of them by The Probing Lensing Anomalies NETwork (PLANET) collaboration and one by a joint effort of five microlensing collaborations (Afonso et al. 2000). The most recent one at the time of writing was described by Albrow et al. (2001). The sensitivity of a linear caustic-crossing event to the brightness profile of the source was recently studied by Gaudi \& Gould (1999) and Rhie \& Bennett (1999). As shown by Rhie \& Bennett (1999), the chromaticity is generally lower than for a single lens. On the other hand, a linear caustic always transits the source completely (with "zero impact parameter"). While single lens transits have the potential to deliver even better results, binary caustic-crossing events are already delivering good results. The approach demonstrated here for the single lens case can be readily applied to the binary case. The linearity of the PCA approach will be particularly beneficial, as the fitting of binary microlensing light curves is computationally heavily burdened by proliferating nonlinear parameters (Albrow et al. 1999b, 2000). This method will substantially improve the analysis of all previously reported as well as upcoming caustic-crossing microlensing events.

\section{SUMMARY}

In this paper we show that limb darkening profiles of source stars in microlensing transit events can be measured by light curve inversion using brightness profile decomposition. Other 
inversion techniques at present fail to reach the accuracy achieved by this method.

Initial tests in $\$ 4.1$ show that under the usual photometric accuracy only two terms of the decomposition can be reasonably recovered. Using linear limb darkening, which suffers from systematic errors, is not ideal. It is necessary to find a limb darkening basis providing in two terms a better description of realistic brightness profiles than does linear limb darkening (LLD). Such a basis can be constructed by principal component analysis of a set of realistic model brightness profiles, as demonstrated in $\S 4.2$. The obtained PCA basis provides the best possible linear description of the used set of profiles, as demonstrated in Appendix B. This method of describing limb darkening should in fact replace the ill-suited and obsolete standard power-law limb darkening model in other practical applications as well.

We demonstrate the described inversion method in $\S 5$ by fully analyzing simulated light curves. In $\S 5.2$ we check the accuracy of recovering the basic lensing parameters. We show that best results are obtained for events with Einstein radii $\epsilon \gtrsim 5$, low impact parameters $p<0.5$ and negligible blend parameters $\beta \ll 1$. As demonstrated in $\S 5.3$, the limb darkening parameter is recovered more reliably using the PCA basis than using the LLD basis, achieving also better brightness profile resolution. The quality of these results is further demonstrated by directly measuring the inversion accuracy, the difference between the recovered and the original brightness profile. The best results are obtained for small impact parameters $p<0.5$. They are only very weakly dependent on the Einstein radius, and independent of the blend. For events with small impact parameters even light curves with $1 \%$ photometry can yield an inversion accuracy of $\pm 2 \%$ from the stellar disk center to $r=0.9$ near the limb using the PCA basis. The accuracy can be further improved by better photometry as well as by increasing the number of measured transit light curve points.

The measured light curves of the microlensing transit event MACHO Alert 95-30 (Alcock et al. 1997) reveal the intrinsic variability of the lensed red giant, as demonstrated in $\S 6$. The time scale, amplitude and irregularity of the variability indicate that the source changes significantly at the light curve peak, and thus its limb darkening cannot be well constrained. This example underscores the need to check the baseline of microlensing transit events for signs of variability as low as $1 \%$ on the event timescale. Inversion results cannot be considered reliable without this safety test.

Binary microlensing events, in which a caustic transits the source star, are much more frequent than the single lens events studied here. Though their sensitivity to the brightness profile of the source tends to be lower, such events have already been used to measure the limb darkening parameters of several stars. The analysis of these caustic-crossing events will be significantly improved by using the PCA approach to limb darkening as described in this work. 
It is a pleasure to thank Dimitar Sasselov for his enthusiastic support, many helpful discussions and comments on the manuscript, as well as for providing the model atmosphere data. The MACHO Alert 95-30 event analysis was made possible by Andy Becker, who kindly supplied the newly reduced light curves. I would like to thank Ramesh Narayan for discussions about inversion techniques, particularly for suggesting to use principal component analysis. Finally I thank the referee, Andy Gould, whose comments and suggestions helped to improve the manuscript.

\section{A. BROADBAND LIGHT CURVE CHROMATICITY}

Following HSL, Figure 8 demonstrates the dependence of the microlensing amplification on the surface brightness profile of the source. In this example a red giant source (effective temperature $\mathrm{T}=3750 \mathrm{~K}$, surface gravity $\log g=0.5$ ) is transited by an $\epsilon=10$ lens at zero impact parameter $p$. BVRI light curves of this event in the lower panel are to be compared with the corresponding normalized brightness profiles in the upper panel (see Appendix B for details). The microlensing amplification clearly traces the shape of the brightness profile. More centrally peaked profiles have more centrally peaked amplification curves (light curves for $p=0$ ). Profiles which have a relatively higher amplification at the source center mostly have a relatively lower amplification close to the source limb, and vice versa.

The sensitivity of microlensing to the brightness profile of the source can be measured by the relative variation of amplification with wavelength, due to the wavelength dependence of the profiles. Following HSL, we define the microlensing chromaticity as the ratio of the total amplification range to the average amplification $\left[A_{\max }-A_{\text {min }}\right] / A_{\text {aver }}$ at a given lens position. The maximum, minimum and average amplifications are evaluated for a particular source over a given spectral range at a given spectral resolution. HSL presented the chromaticity of low and high resolution spectra, here we demonstrate the broadband chromaticity using merely the four profiles from Figure 8. Plotted as a function of lens position in Figure 9, the chromaticity curve has the same character as those presented in HSL with a slightly lower amplitude due to the lower resolution. The individual curves correspond to lenses with different Einstein radii. Note that the chromaticity becomes independent of $\epsilon$ for

$\epsilon \gtrsim 5$. The highest sensitivity to limb darkening occurs when the lens is close to the source center, another somewhat less sensitive region is close to the source limb. The sensitivity drops rapidly for non-transit lens positions. These overall features were first reported in the works of Witt (1995); Simmons, Newsam, \& Willis (1995); Valls-Gabaud (1995); Bogdanov \& Cherepashchuk (1995) and Gould \& Welch (1996).

In the vicinity of $l \simeq 0.73$ the amplification is largely achromatic, i.e., independent of 
the underlying limb darkening profile. The occurrence of this achromatic point is a direct consequence of the narrowly confined region around $r \simeq 0.69$ where all the normalized brightness profiles in the upper panel of Figure 8 intersect. As Figure 2 and even the higher resolution results in HSL demonstrate, this narrow intersection region is common to all the computed profiles (see also Appendix B). It can be shown that this property is an indication that the full range of profiles is well modelled by a linear combination of two radial functions. Any such a suitable model has an exact intersection point common to all the profiles. The position of this point, however, depends on the particular model. As a consequence, using a limb darkening model with a wrong intersection point in the analysis of microlensing light curves will additionally result in inaccurate values of the inferred microlensing parameters. However, as mentioned in $\S 7$ such a wrong model could be told by the pattern of residuals of the light-curve fit. See Appendix B for more details on limb darkening profiles.

\section{B. LIMB DARKENING PROFILES AND THEIR FITS}

In this work we used limb darkening profiles obtained from eight model atmospheres of cool red giants computed by Dimitar Sasselov (see HSL for details). The effective temperatures and surface gravities of these models are $\mathrm{T} \in\{3500 \mathrm{~K}, 3750 \mathrm{~K}, 4000 \mathrm{~K}\}$ and $\log g \in\{0$, $0.5,1.0\}$. Specific intensity was computed for 17 rays passing through the atmosphere at different positions on the projected disk. These positions are marked in Figure 2. The $B V R I$ profiles were obtained from these results using the filter response functions provided by Bessell (1990). To obtain a full radial profile, these 17 points were interpolated using a cubic spline with boundary conditions $B^{\prime}(0)=0$ and $B^{\prime \prime}(1)=0{ }^{19}$. The variation of the profiles in the studied physical range is strongest with wavelength (filter), weaker with temperature and weakest with surface gravity. All profiles with $\log g=0.5$ are plotted in Figure 2, normalized to unit total flux. Two trends are obvious: the profiles at longer wavelengths are flatter; so are mostly the profiles of higher temperature models in the studied range.

The two limb darkening models used in this paper (PCA, LLD) are demonstrated in Figure 10. The PCA basis was obtained directly by principal component analysis of all 32 computed model profiles, as described in $§ 4.2$. The standard linear limb darkening (LLD) basis is defined by equation (22). Figure 10 compares the full ranges of profiles covered by the two models, normalized to unit flux and plotted on the same scale as Figure 2. The ranges are limited physically by requiring positiveness and a central peak for the profile. Notice

\footnotetext{
${ }^{19}$ The latter condition is fairly arbitrary, but has no global effect on the quality of the interpolation (see Fig. 2) due to the high number of computed points constraining the profile close to the limb.
} 
that the LLD model is particularly ill-suited for describing the peaked $B$ profiles in Figure 2, as well as the behavior close to the limb in general. Also note that LLD profiles with low values of the parameter $\kappa=\alpha_{2} / \alpha_{1}$ are unrealistically flat. Finally, there is a difference in the position of the intersection point as discussed in Appendix A. The LLD point is $\gtrsim 0.05$ closer to the limb than the PCA point, which lies directly in the narrow intersection region of the profiles in Figure 2. As a result, simulated microlensing light curves generated using LLD will intersect at $l \simeq 0.77$, those generated using PCA at $l \simeq 0.73$. This is the probable cause for the large systematic error in the source radius crossing time $t_{*}$ recovered from microlensing light curves using LLD in $§ 5.2$.

The difference in the quality of least-squares fits ${ }^{20}$ to all the 32 computed profiles using the two limb darkening models is demonstrated by the results in Table 1 . The columns of the table contain for both limb darkening models the best-fit limb darkening parameter $\kappa=\alpha_{2} / \alpha_{1}$, the normalized root-mean-square deviation of the fit,

$$
\delta_{f i t}=\left\{\int\left[B(r)-\alpha_{1} f_{1}(r)-\alpha_{2} f_{2}(r)\right]^{2} d r / \int B^{2}(r) d r\right\}^{1 / 2},
$$

and the relative flux excess $\Delta F / F$ of the best-fit solution over the original profile. The PCA fits always leave an r.m.s. deviation $\delta_{f i t} \lesssim 0.7 \%$, while the LLD fits can leave a deviation of as much as 3\% (in the case of the B profiles). The LLD fits are marginally better than the PCA fits only for some of the R3750 and R4000 profiles. The more striking difference is in the excess flux. While the fluxes of the PCA fits differ from the actual source fluxes by less than $2 \times 10^{-4}$, the LLD fits overestimate the source flux for all B, V, R3500 and R3750 profiles, by as much as $0.75 \%$. This renders the LLD model also less useful for fitting microlensing light curves, where such a difference is not negligible. In the remaining profiles the flux is underestimated; a flux accuracy comparable to the PCA fits is never achieved.

Although our PCA basis was constructed from limb darkening profiles of cool giant models, Figure 11 demonstrates this PCA model can be better suited than the LLD model even for fitting measured data of narrow-band solar limb darkening. For this figure we used data published by Mitchell (1959) spanning the BVRI spectral range, namely his measurements at 450,545, 660 and $848 \mathrm{~nm}$. The radial coordinates of the data points are marked in the figure by crosses. In order to give even weight to all radii ${ }^{21}$, we interpolated the observed data by a spline in the manner described at the beginning of this appendix to obtain $B(r)$. We performed least-squares fits to these interpolations using both limb darkening models and plotted the deviation curves $B(r) / B_{f i t}(r)-1$. The small-amplitude bumps in the curves are due to the interpolation. As seen from the figure, while for $450 \mathrm{~nm}$

\footnotetext{
${ }^{20}$ Computed as unweighted fits to the spline-interpolated profiles (see Fig. 2).

${ }^{21}$ Note that the narrow region close to the limb has the highest density of data points.
} 
and $848 \mathrm{~nm}$ both models have a comparable maximum deviation (within the range $r \lesssim 0.9$ ), for $545 \mathrm{~nm}$ and $660 \mathrm{~nm}$ the PCA model fits the data better. It is interesting to note that here the LLD model systematically provides fits more peaked than the original profiles.

A more rigorous approach for studying different stellar types would be to construct PCA bases from model atmospheres of such stars. The reason for the cool giant PCA basis fitting the solar data is primarily physical. For both stellar types as well as for a wide range of other cool stars, the dominant source of opacity determining the limb darkening in the optical range is the same - namely, the $\mathrm{H}^{-}$ion (e.g., Gray 1992). 


\section{REFERENCES}

Afonso, C., et al. 2000, ApJ, 532, 340

Albrow, M. D., et al. 1999a, ApJ, 522, 1011

Albrow, M. D., et al. 1999b, ApJ, 522, 1022

Albrow, M. D., et al. 2000, ApJ, 534, 894

Albrow, M. D., et al. 2001, ApJ, 549, 759

Alcock, C., et al. 1997, ApJ, 491, 436

An, J. H., et al. 2002, ApJ, 572, 521

Becker, A., et al. 1997, BAAS, 29, 1347

Bessell, M. S. 1990, PASP, 102, 1181

Bogdanov, M. B., \& Cherepashchuk, A. M. 1995, Astron. Rep., 39, 779

Bogdanov, M. B., \& Cherepashchuk, A. M. 1996, Astron. Rep., 40, 713

Bogdanov, M. B., \& Cherepashchuk, A. M. 1999, Astron. Rep., 43, 601

Brown, T. M., Charbonneau, D., Gilliland, R. L., Noyes, R. W., \& Burrows, A. 2001, ApJ, 552,699

Bryce, H. M., \& Hendry, M. A. 2000, to appear in ASP Conf. Ser., Microlensing 2000, ed. J. W. Menzies \& P. D. Sackett (San Francisco: ASP), preprint (astro-ph/0004250)

Burns, D., et al. 1997, MNRAS, 290, L11

Byrd, P. F., \& Friedman, M. D. 1971, Handbook of Elliptic Integrals for Engineers and Scientists (2nd ed.; Berlin: Springer)

Craig, I. J. D., \& Brown, J. C. 1986, Inverse Problems in Astronomy (Bristol: Adam Hilger)

Cummings, I. N., Hearnshaw, J. B., Kilmartin, P. M., \& Gilmore, A. C. 1998, in ASP Conf. Ser. 135, A Half-Century of Stellar Pulsation Interpretations, ed. P. A. Bradley \& J. A. Guzik (San Francisco: ASP), 213

Deeg, H. J., Garrido, R., \& Claret, A. 2001, New Astronomy, 6, 51

Gaudi, B. S., \& Gould, A. 1999, ApJ, 513, 619 
Gaudi, B. S., \& Sackett, P. D. 2000, ApJ, 528, 56

Gould, A. 2000, ApJ, 542, 785

Gould, A., \& Welch, D. L. 1996, ApJ, 464, 212

Gray, D. F. 1992, The Observation and Analysis of Stellar Photospheres (2nd ed.; Cambridge: Cambridge University Press)

Gray, N., \& Coleman, I. J. 2000, to appear in ASP Conf. Ser., Microlensing 2000, ed. J. W. Menzies \& P. D. Sackett (San Francisco: ASP), preprint (astro-ph/0004200)

Griest, K., \& Hu, W. 1992, ApJ, 397, 362

Griest, K., \& Safizadeh, N. 1998, ApJ, 500, 37

Grygar, J., Cooper, M. L., \& Jurkevich, I. 1972, Bull. Astr. Inst. Cz., 23, 147

Hajian, A. R., et al. 1998, ApJ, 496, 484

Han, C., \& Gould, A. 1997, ApJ, 480, 196

Han, C., \& Jeong, Y. 1998, MNRAS, 301, 231

Hendry, M. A., Coleman, I. J., Gray, N., Newsam, A. M., \& Simmons, J. F. L. 1998, NewAR, 42, 125

Heyrovský, D., \& Loeb, A. 1997, ApJ, 490, 38

Heyrovský, D., \& Sasselov, D. 2000, ApJ, 529, 69

Heyrovský, D., Sasselov, D., \& Loeb, A. 2000, ApJ, 543, 406 (HSL)

Jacob, A. P., Bedding, T. R., Robertson, J. G., \& Scholz, M. 2000, MNRAS, 312, 733

Jha, S., Charbonneau, D., Garnavich, P. M., Sullivan, D. J., Sullivan, T., Brown, T. M., \& Tonry, J. L. 2000, ApJ, 540, L45

Jolliffe, I. T. 1986, Principal Component Analysis (New York: Springer)

Jorissen, A., Mowlavi, N., Sterken, C., \& Manfroid, J. 1997, A\&A, 324, 578

Koen, C., \& Laney, D. 2000, MNRAS, 311, 636

Loeb, A., \& Sasselov, D. 1995, ApJ, 449, L33 
Mitchell, W. E. 1959, ApJ, 129, 93

Mozurkewich, D., et al. 1991, AJ, 101, 2207

Paczyński, B. 1996, ARA\&A, 34, 419

Percy, J. R., \& Parkes, M. 1998, PASP, 110, 1431

Percy, J. R., \& Polano, S. 1998, in ASP Conf. Ser. 135, A Half-Century of Stellar Pulsation Interpretations, ed. P. A. Bradley \& J. A. Guzik (San Francisco: ASP), 249

Popper, D. M. 1984, AJ, 89, 132

Popper, D. M., Andersen, J., Clausen, J. V., \& Nordström, B. 1985, AJ, 90, 1324

Preisendorfer, R. W. 1988, Principal Component Analysis in Meteorology and Oceanography (Amsterdam: Elsevier)

Press, W. H., Teukolsky, S. A., Vetterling, W. T., \& Flannery, B. P. 1992, Numerical Recipes in C (2nd ed.; Cambridge: Cambridge University Press)

Quirrenbach, A., Mozurkewich, D., Buscher, D. F., Hummel, C. A., \& Armstrong, J. T. 1996, A\&A, 312, 160

Rhie, S. H., et al. 1999, ApJ, 522, 1037

Rhie, S. H., \& Bennett, D. P. 1999, ApJ, submitted, preprint (astro-ph/9912050)

Sasselov, D. D. 1996, in ASP Conf. Ser. 109, Cool Stars 9, ed. R. Pallavicini \& A. K. Dupree (San Francisco: ASP), 541

Simmons, J. F. L., Newsam, A. M., \& Willis, J. P. 1995, MNRAS, 276, 182

Uitenbroek, H., Dupree, A. K., \& Gilliland, R. L. 1998, AJ, 116, 2501

Valls-Gabaud, D. 1995, in Large Scale Structure in the Universe, ed. J. P. Mücket, S. Gottlöber, \& V. Müller (Singapore: World Scientific), 326

Witt, H. J. 1995, ApJ, 449, 42 


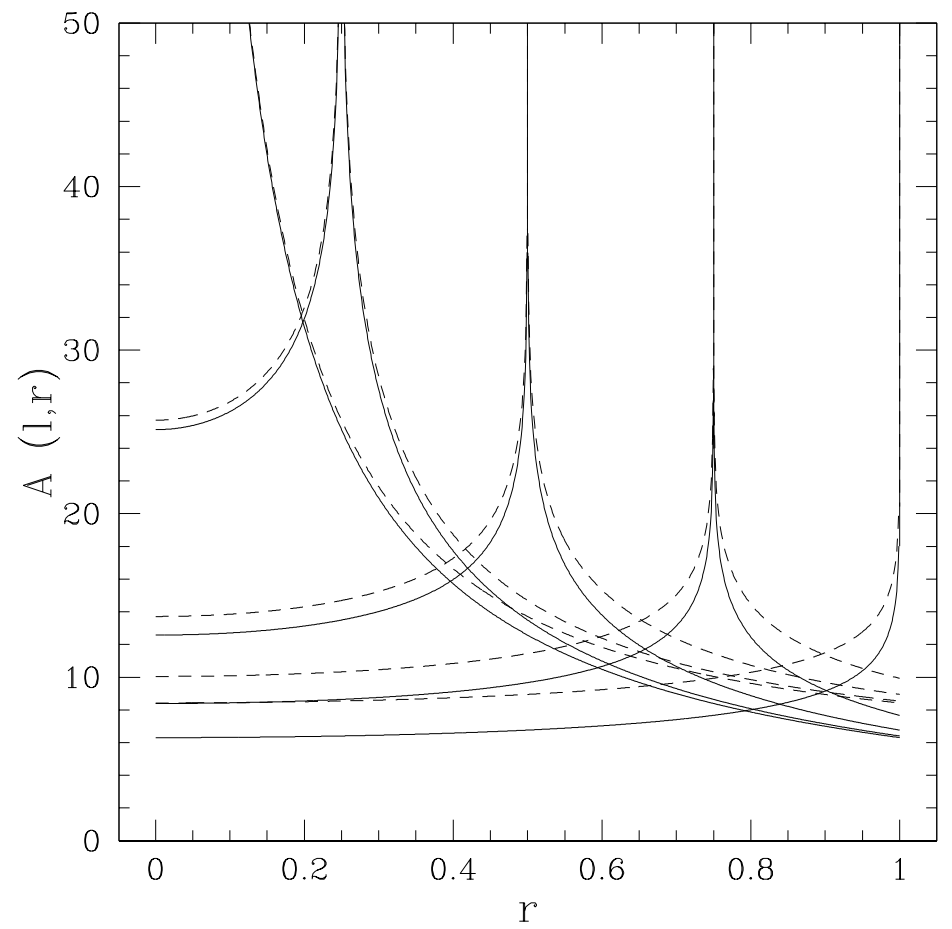

Fig. 1.- Dashed line: Angle-integrated amplification $\mathcal{A}(l, r)$ for a lens with Einstein radius $\epsilon=1$ as a function of radial distance $r$ for lens positions $l=0,0.25,0.5,0.75$ and 1 (curves with peaks from left to right). Solid line: $\mathcal{A}(l, r) / \epsilon$ for a lens with $\epsilon=10$, same lens positions. 


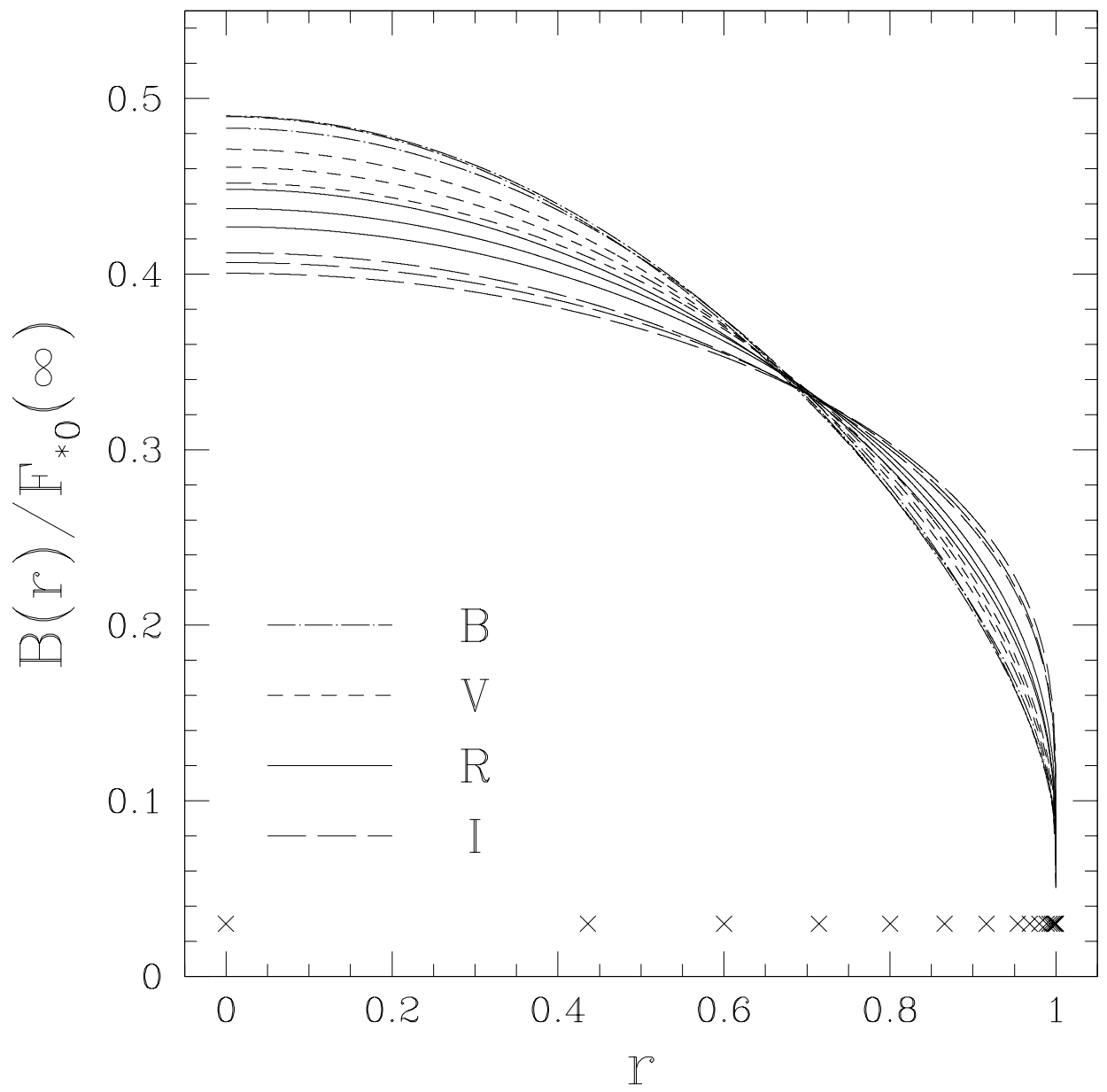

Fig. 2.- Normalized BVRI brightness profiles of three $\log g=0.5$ model atmospheres (from upper to lower at $r=0$ in each filter): $\mathrm{T}=3500,3750,4000 \mathrm{~K}$. Radial positions of computed points are marked at bottom, plotted curves are spline interpolations to these points. 


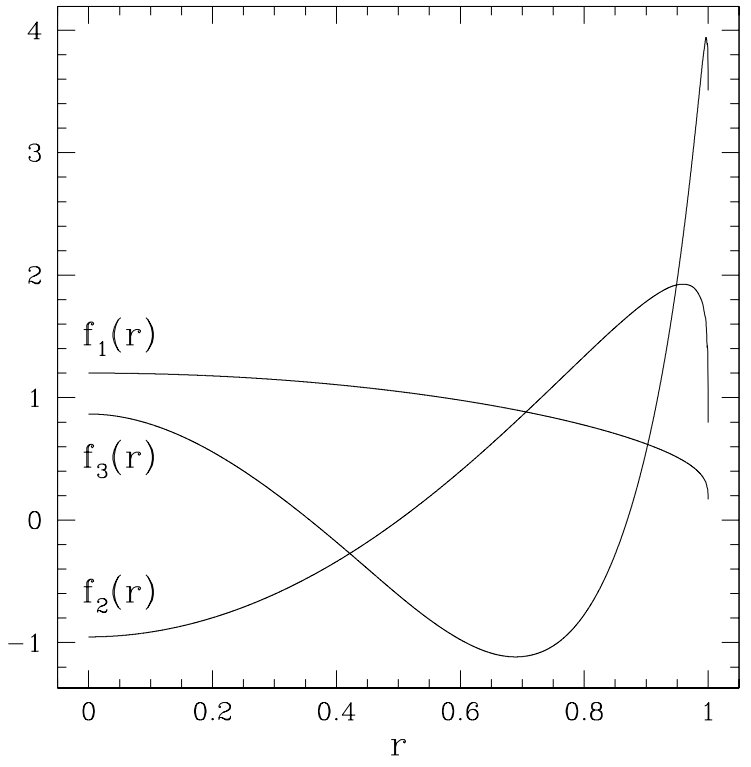

Fig. 3.- First three orthonormal functions of the PCA basis for brightness profile decomposition, obtained by principal component analysis of 32 brightness profiles of red giant model atmospheres (see Table 1). 

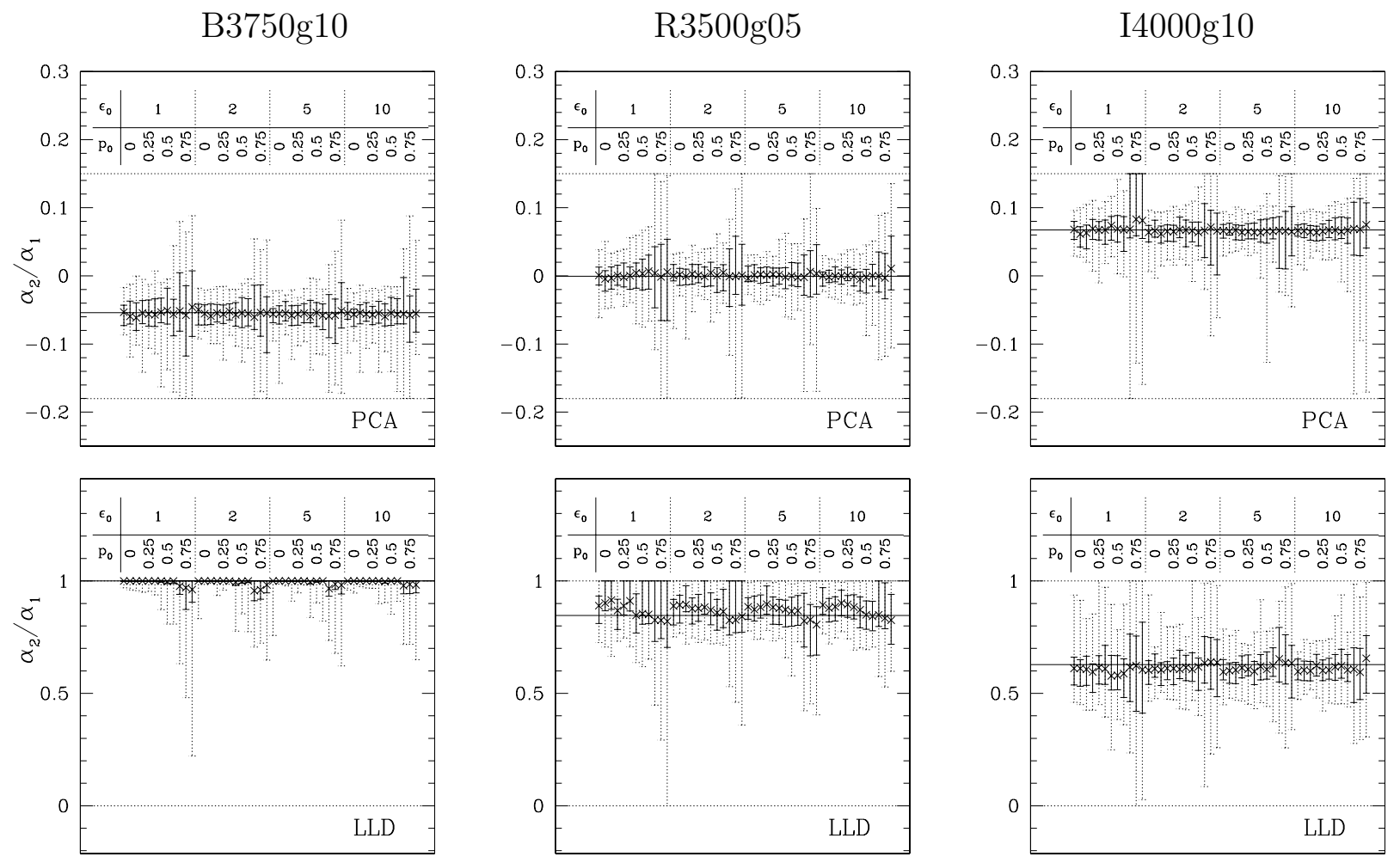

Fig. 4.- Recovered PCA (upper panels) and LLD (lower panels) limb darkening parameters for model profiles: B3750g10 (left column), R3500g05 (central column) and I4000g10 (right column). Individual points correspond to values of the original Einstein radius $\epsilon_{0}$ and impact parameter $p_{0}$ directly above them in the table, triplets of neighboring points correspond to blend parameters $\beta_{0}=0,0.25,0.5$. Dotted vertical bars: range of values from all simulations. Solid horizontal lines: original best-fit values. Dotted horizontal lines: full parameter range. 

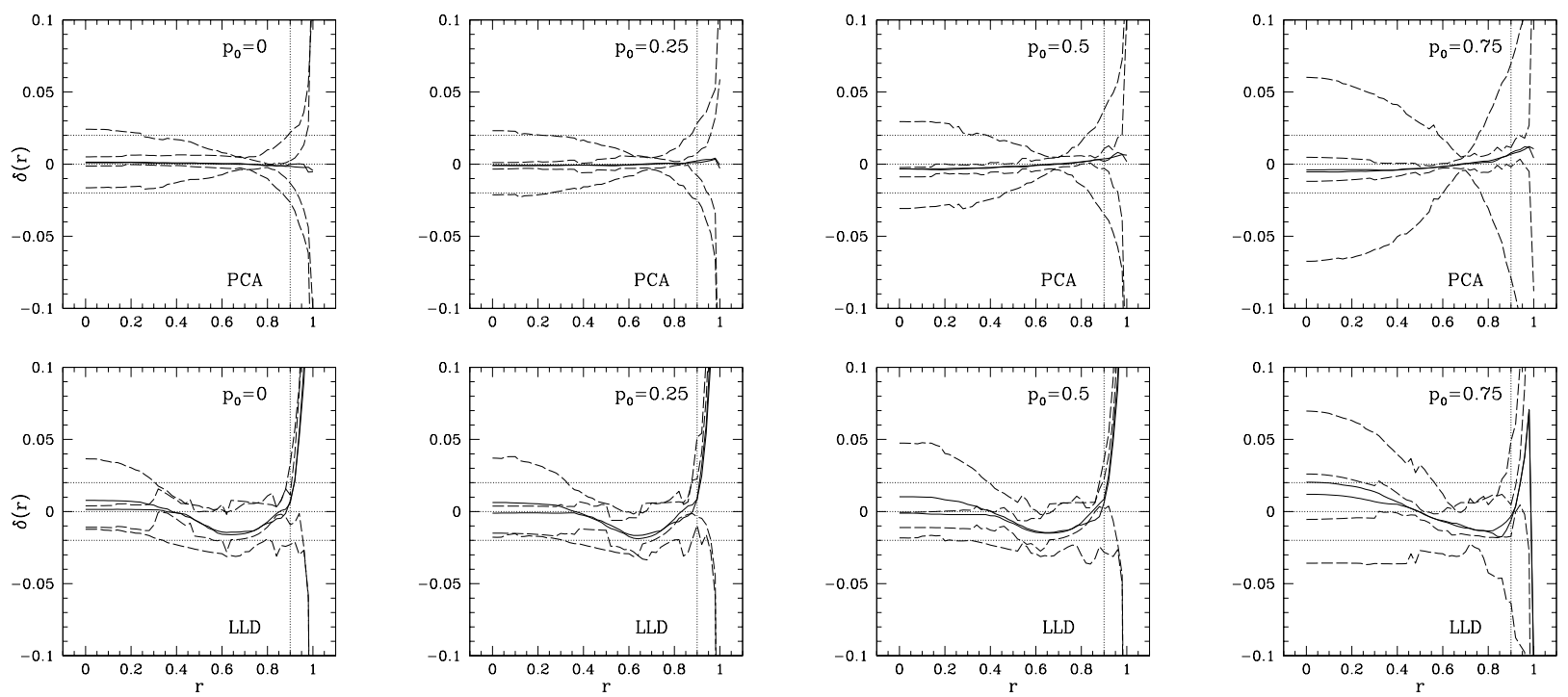

Fig. 5.- Brightness profile inversion accuracy from center to limb of lensed star, for $\epsilon_{0}=10$. Bold solid line: bias $\delta_{B}$; thin solid line: "median median" $\delta_{M M}$; bold dashed lines: total errorbars; thin dashed lines: systematic errorbars. Dotted lines: $\delta=0, \pm 2 \%$ and $r=0.9$. Multiply total errorbars by inverted profile to obtain absolute errorbars. Columns correspond to marked impact parameters $p_{0}$. Upper panels: PCA inversion; lower panels: LLD inversion. 

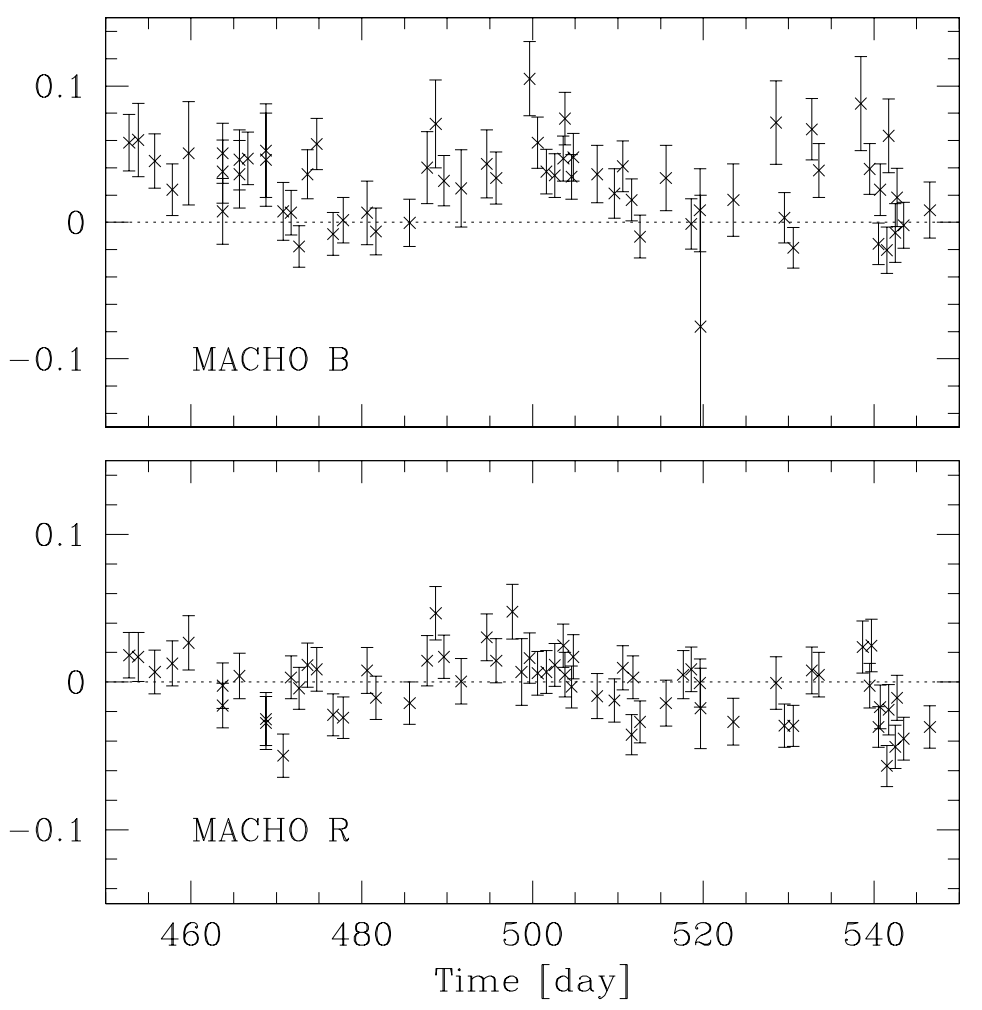

Fig. 6.- Baseline detail of the MACHO B (upper panel) and MACHO R (lower panel) light curves of the MACHO Alert 95-30 event. Vertical axes correspond to relative flux deviations from the best-fit microlensing solution baselines (marked by dotted lines). Note the correlated variability as well as the systematically positive MACHO B deviation. Following Alcock et al. (1997), time is measured from JD 2,448,623.50 . 


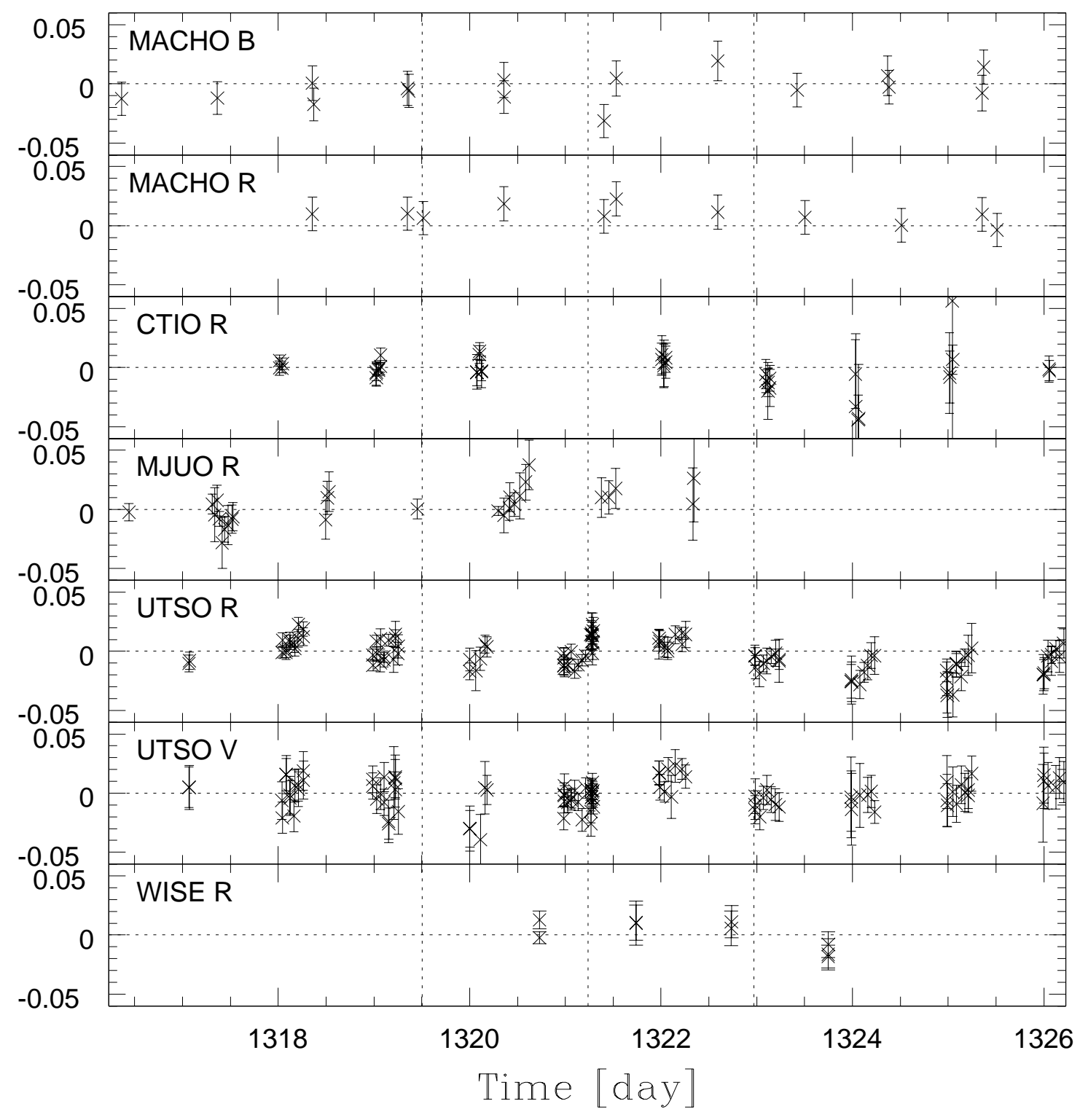

Fig. 7.- Relative residuals $F_{i} / F\left(l_{i}\right)-1$ of the best-fit microlensing solution close to the peak of the MACHO Alert 95-30 event. Individual panels correspond to light curves marked in the left corners. The time of closest approach $t_{0}$ and limb crossing times of the best-fit solution are marked by vertical dotted lines (time is measured from JD 2,448,623.50) . 

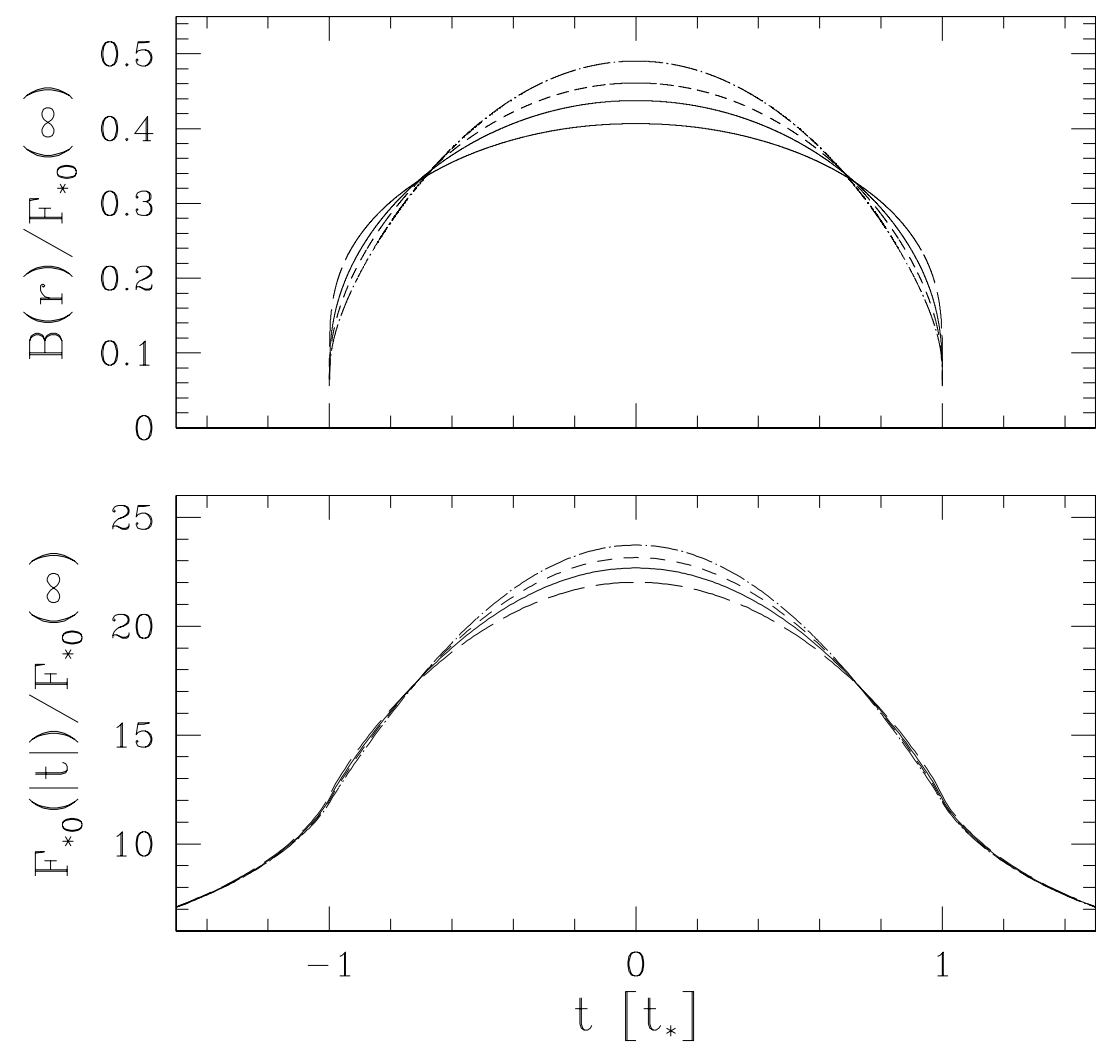

Fig. 8.- Lower panel: BVRI amplification light curves (from top to bottom at $t=0$ ) of a microlensing transit of a $\mathrm{T}=3750 \mathrm{~K}, \log g=0.5 \mathrm{red}$ giant by an $\epsilon=10$ lens with a zero impact parameter. Upper panel: corresponding brightness profiles normalized to unit flux (same sequence at $t=0$ ), horizontal scale matches the lens position. 


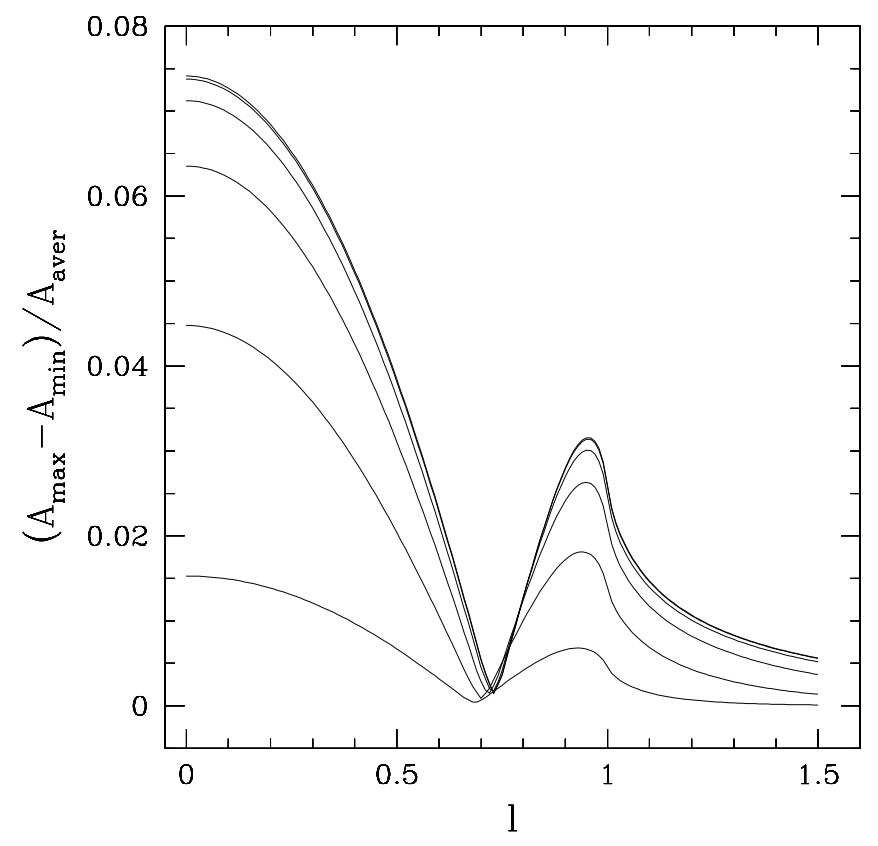

Fig. 9.- Broadband microlensing chromaticity: relative variation of microlensing amplification from Figure 8 with filter passband, as a function of lens position (in source radius units). Curves correspond to Einstein radii $\epsilon=0.2,0.5,1,2,5,10$ (from lowest curve). 

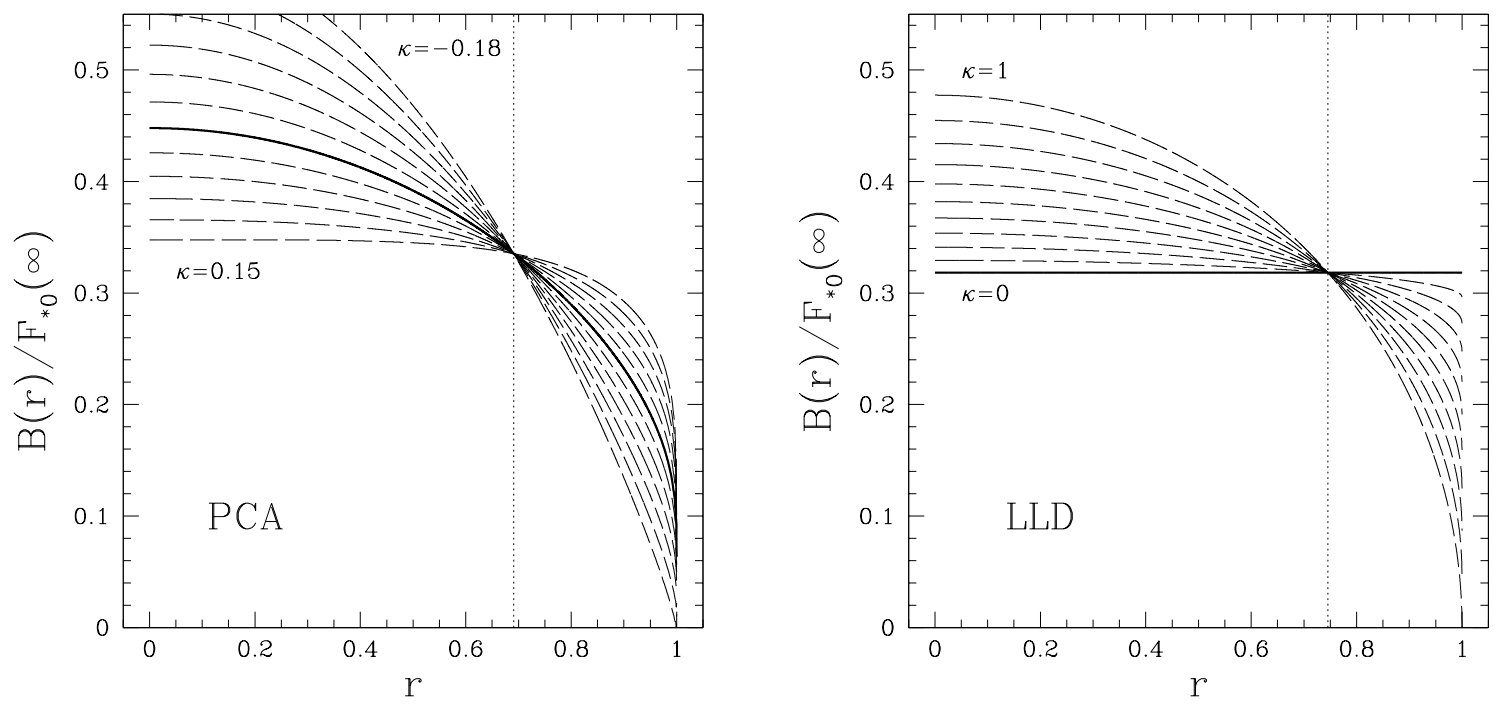

Fig. 10.- Full parameter range of limb darkening profiles using the 2-term PCA (left panel) and LLD bases (right panel), normalized to unit flux and plotted on same scale as Figure 2. Limb darkening parameter values $\kappa$ are spaced by 0.03 in the PCA model, by 0.1 in the LLD model. Solid curves correspond to $\kappa=0$ in each model (first basis term). The vertical dotted line marks the common intersection point, characteristic of each model. 

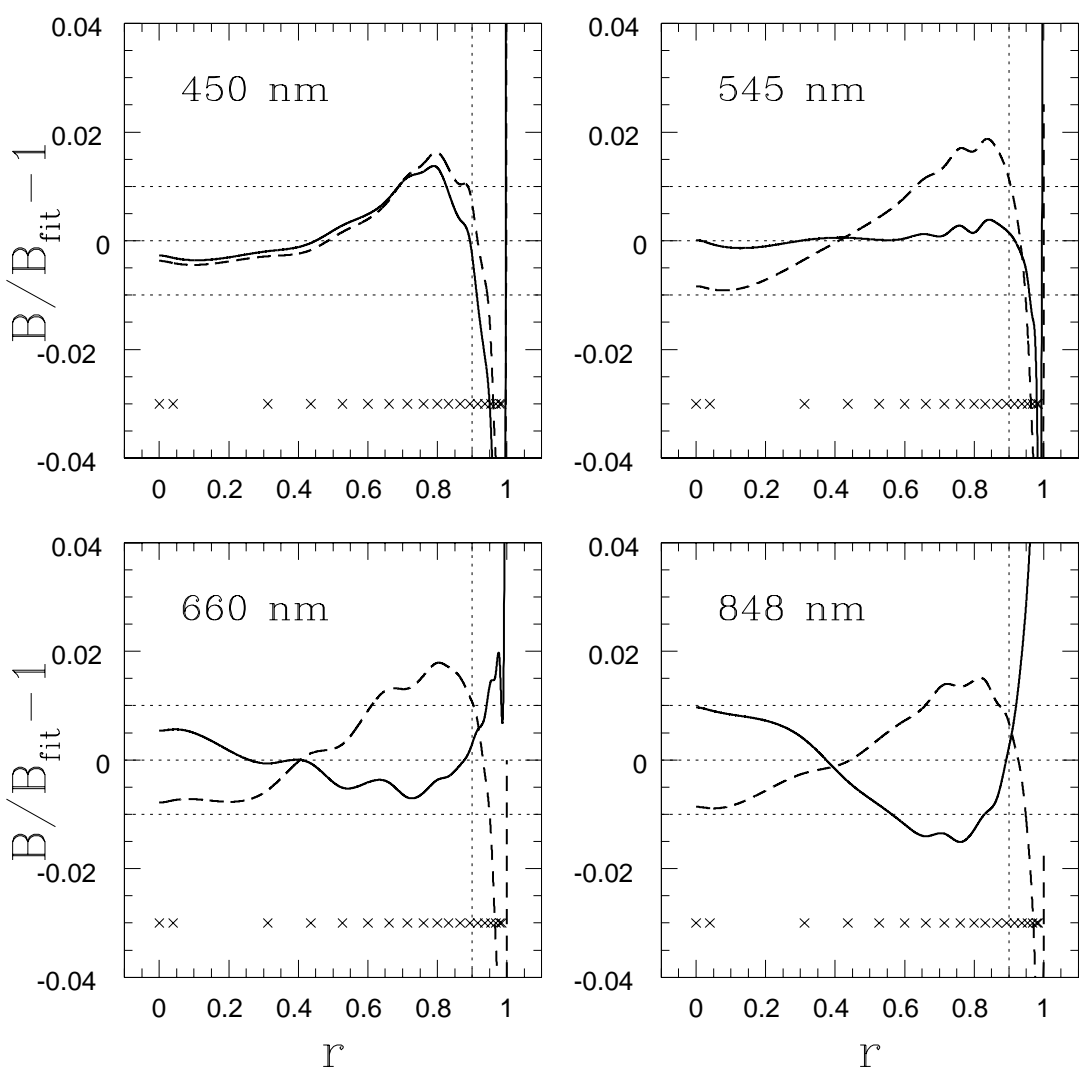

Fig. 11. - Comparison of PCA (solid line) and LLD (dashed line) residuals of fits to measured solar limb darkening data (Mitchell 1959) at four selected wavelengths as denoted. Radial positions of the data points are marked at the lower edge of the plots. Dotted lines serve for orientation: $B / B_{f i t}-1=0, \pm 1 \%$ and $r=0.9$. 


\begin{tabular}{|c|c|c|c|c|c|c|}
\hline \multirow[t]{2}{*}{ Profile } & \multicolumn{3}{|c|}{$\overline{\text { PCA fit }}$} & \multicolumn{3}{|c|}{$\overline{\text { LLD fit }}$} \\
\hline & $\begin{array}{c}\text { Parameter } \\
{\left[10^{-2}\right]}\end{array}$ & $\begin{array}{c}\text { Deviation } \\
{\left[10^{-2}\right]}\end{array}$ & $\begin{array}{c}\text { Excess flux } \\
{\left[10^{-4}\right]}\end{array}$ & Parameter & $\begin{array}{c}\text { Deviation } \\
{\left[10^{-2}\right]}\end{array}$ & $\begin{array}{c}\text { Excess flux } \\
{\left[10^{-4}\right]}\end{array}$ \\
\hline B3500g00 & -4.46 & 0.68 & 0.61 & 0.970 & 2.98 & 74.61 \\
\hline B3500g05 & -4.96 & 0.56 & 1.06 & 0.985 & 3.00 & 75.32 \\
\hline B3500g10 & -5.18 & 0.44 & 1.52 & 0.993 & 2.94 & 73.96 \\
\hline B3750g00 & -4.67 & 0.47 & -1.84 & 0.977 & 2.85 & 69.68 \\
\hline B3750g05 & -5.19 & 0.33 & -1.48 & 0.993 & 2.87 & 70.47 \\
\hline B3750g10 & -5.41 & 0.23 & -1.06 & 1.000 & 2.85 & 70.00 \\
\hline B4000g05 & -4.51 & 0.19 & -1.41 & 0.977 & 2.30 & 55.46 \\
\hline $\mathrm{B} 4000 \mathrm{~g} 10$ & -4.84 & 0.25 & -1.09 & 0.988 & 2.29 & 55.09 \\
\hline V3500g00 & -2.89 & 0.12 & 0.74 & 0.931 & 1.81 & 44.15 \\
\hline V3500g05 & -2.99 & 0.14 & 0.97 & 0.934 & 1.83 & 44.55 \\
\hline V3500g10 & -2.96 & 0.14 & 1.14 & 0.933 & 1.83 & 44.83 \\
\hline V3750g00 & -1.75 & 0.27 & -0.65 & 0.898 & 1.32 & 30.96 \\
\hline V3750g05 & -1.83 & 0.32 & -0.31 & 0.901 & 1.28 & 30.31 \\
\hline V3750g10 & -1.79 & 0.33 & -0.12 & 0.900 & 1.26 & 29.81 \\
\hline V4000g05 & -0.75 & 0.51 & -0.46 & 0.871 & 0.79 & 18.21 \\
\hline V4000g10 & -0.77 & 0.53 & -0.26 & 0.872 & 0.78 & 17.86 \\
\hline R3500g00 & -0.25 & 0.23 & 0.94 & 0.853 & 0.94 & 22.06 \\
\hline R3500g05 & -0.06 & 0.11 & 0.73 & 0.847 & 1.02 & 24.02 \\
\hline R3500g10 & 0.21 & 0.11 & 0.53 & 0.837 & 1.07 & 25.36 \\
\hline R3750g00 & 1.32 & 0.46 & 0.91 & 0.808 & 0.31 & 5.41 \\
\hline R3750g05 & 1.25 & 0.42 & 0.72 & 0.810 & 0.34 & 6.59 \\
\hline R3750g10 & 1.21 & 0.35 & 0.38 & 0.810 & 0.39 & 8.30 \\
\hline R4000g05 & 2.68 & 0.35 & 0.37 & 0.765 & 0.26 & -1.54 \\
\hline $\mathrm{R} 4000 \mathrm{~g} 10$ & 2.68 & 0.35 & 0.32 & 0.765 & 0.26 & -1.39 \\
\hline I3500g00 & 4.78 & 0.16 & 0.15 & 0.694 & 0.48 & -3.97 \\
\hline $\mathrm{I} 3500 \mathrm{~g} 05$ & 5.04 & 0.28 & -0.10 & 0.685 & 0.46 & -2.78 \\
\hline I3500g10 & 5.30 & 0.38 & -0.27 & 0.675 & 0.46 & -2.24 \\
\hline I3750g00 & 5.77 & 0.09 & 0.28 & 0.663 & 0.74 & -11.77 \\
\hline I3750g05 & 5.79 & 0.16 & -0.16 & 0.662 & 0.66 & -10.40 \\
\hline $\mathrm{I} 3750 \mathrm{~g} 10$ & 5.80 & 0.25 & -0.65 & 0.660 & 0.58 & -8.80 \\
\hline I4000g05 & 6.74 & 0.33 & -0.65 & 0.629 & 0.77 & -12.94 \\
\hline $\mathrm{I} 4000 \mathrm{~g} 10$ & 6.76 & 0.36 & -0.88 & 0.628 & 0.74 & -12.42 \\
\hline
\end{tabular}

Table 1: List of model brightness profiles used in this work. Adopted profile names indicate the filter, effective temperature and surface gravity of the model atmosphere. Following columns: best-fit PCA and LLD limb darkening parameters $\kappa$, normalized r.m.s. deviations $\delta_{f i t}$ and relative excess fluxes $\Delta F / F$ of the fits. 